\title{
Radio, millimeter and optical monitoring of GRB 030329 afterglow: constraining the double jet model ${ }^{\star}$
}

\author{
L. Resmi ${ }^{1,2}$, C. H. Ishwara-Chandra ${ }^{3}$, A. J. Castro-Tirado ${ }^{4}$, D. Bhattacharya ${ }^{1}$, A. P. Rao ${ }^{3}$, M. Bremer $^{5}$, \\ S. B. Pandey $^{6}$, D. K. Sahu ${ }^{7,8}$, B. C. Bhatt ${ }^{7}$, R. Sagar ${ }^{6}$, G. C. Anupama ${ }^{7}$, A. Subramaniam ${ }^{7}$, A. Lundgren ${ }^{9}, 10$, \\ J. Gorosabel ${ }^{4}$, S. Guziy ${ }^{4,11}$, A. de Ugarte Postigo ${ }^{4}$, J. M. Castro Cerón ${ }^{12}$, and T. Wiklind ${ }^{12}$ \\ 1 Raman Research Institute, Bangalore 560080, India \\ e-mail: resmi@rri.res.in \\ 2 Joint Astronomy Programme, Indian Institute of Science, Bangalore 560012, India \\ 3 National Center for Radio Astrophysics, Post Bag 3, Ganeshkhind, Pune 411007, India \\ ${ }^{4}$ Instituto de Astrofísica de Andalucía, Apartado de Correos, 3.004, 18080 Granada, Spain \\ 5 IRAM - Institut de Radio Astronomie Millimétrique, 300 rue de la Piscine, 38406 Saint-Martin d'Hères, France \\ ${ }^{6}$ Aryabhatta Research Institute of Observational Sciences, Manora Peak, Naini Tal 263129, India \\ 7 Indian Institute of Astrophysics, Bangalore 560034, India \\ 8 Center for Research \& Education in Science \& Technology, Hosakote, Bangalore 562114, India \\ 9 European Southern Observatory, Alonso de Córdova, Casilla 19001, Chile \\ 10 Stockholm Observatory, 10691 Stockholm, Sweden \\ 11 Astronomical Observatory, Nikolaev State University, Nikolskaja, 24, Nikolaev 54030, Ukraine \\ 12 Space Telescope Science Institute, 3.700 San Martín Dr., Baltimore, MD 21.218-2.463, USA
}

Received 12 July 2004 / Accepted 12 April 2005

\begin{abstract}
We present radio, millimeter and optical observations of the afterglow of GRB $030329 . U B V R_{\mathrm{C}} I_{\mathrm{C}}$ photometry is presented for a period of $3 \mathrm{~h}$ to 34 days after the burst. Radio monitoring at $1280 \mathrm{MHz}$ has been carried out using the GMRT for more than a year. Simultaneous millimeter observations at $90 \mathrm{GHz}$ and $230 \mathrm{GHz}$ have been obtained from the Swedish-ESO Submillimeter Telescope (SEST) and the IRAM-PdB interferometer over more than a month following the burst. We use these data to constrain the double jet model proposed by Berger et al. (2003) for this afterglow. We also examine whether instead of the two jets being simultaneously present, the wider jet could result from the initially narrow jet, due to a fresh supply of energy from the central engine after the "jet break".
\end{abstract}

Key words. gamma rays: bursts

\section{Introduction}

The Gamma Ray Burst of 29th March 2003 has been an unique event. At a distance of $\sim 870 \mathrm{Mpc}$ (assuming a cosmology of $\Omega_{\Lambda}=0.7$ and $\Omega_{\mathrm{m}}=0.3$, and a redshift of 0.1685 (Greiner et al. 2003a) it is the second nearest GRB for which an afterglow has been observed. The optical and radio afterglow of this burst has been one of the brightest detected till date (Peterson \& Price 2003). The spectral signature of a supernova (SN2003dh) emerged in the optical transient a few days after the burst (Stanek et al. 2003) and thus provided the first unambiguous evidence of the long suspected association between Gamma Ray Bursts and Supernovae. Multifrequency observations indicated that the GRB consisted of at least two jet-like components of ejection with different opening angles and Lorentz

* Table 2 is only available in electronic form at http://www. edpsciences.org factors, in addition to the supernova component (Berger et al. 2003).

GRB 030329 was detected and localized by the HETE-II satellite (Vanderspek et al. 2003a). The trigger H2652 occurred on 29th March 2003, at UT 11:37:14.7 and lasted more than $100 \mathrm{~s}$. This was one of the brightest bursts detected by the instrument, with a 30-400 KeV fluence of $1.1 \times 10^{-4} \mathrm{erg} \mathrm{cm}^{-2}$. The Soft X-ray Camera on board HETE - II localized the burst to be at RA $(\mathrm{J} 2000)=10^{\mathrm{h}} 44^{\mathrm{m}} 49^{\mathrm{s}}$ and Dec $(\mathrm{J} 2000)=$ $+21^{\circ} 28^{\prime} 44^{\prime \prime}$ within an error circle of radius 2 arcmin. The temporal profile showed two distinct peaks in the burst, separated by $\sim 11 \mathrm{~s}$. Fluence in the lower energy band, $S_{(7-30 \mathrm{KeV})}$, was $5.5 \times 10^{-5} \mathrm{erg} \mathrm{cm}^{-2}$, which implies a hardness ratio of $S_{(7-30 \mathrm{KeV})} / S_{(30-400 \mathrm{KeV})}>0.33$, classifying this GRB into the "X-ray rich" category (Vanderspek et al. 2004). In a worldwide observational campaign, the afterglow was detected in all possible wavebands. The first X-ray detection by RXTE $\sim \mathrm{h}$ after the burst found the source to be extremely bright with 
a $2-10 \mathrm{KeV}$ flux of $1.4 \times 10^{-10} \mathrm{erg} \mathrm{cm}^{-2} \mathrm{~s}^{-1}$ (Marshall \& Swank 2003). The optical transient had an $R$-band magnitude $\sim 12$ when it was reported by Peterson \& Price (2003) and Torii (2003). The VLA detected a bright $3.5 \mathrm{mJy}$ radio afterglow at $8.46 \mathrm{GHz}$ (Berger et al. 2003) on 2003 March 30.06 UT. Later follow up observations in other radio frequencies were reported by Pooley et al. (2003), Rao et al. (2003a,b), Hoge et al. (2003) and Kuno et al. (2003). Around 7 days after the burst the optical spectrum showed the signature of an underlying supernova emission (Stanek et al. 2003; Hjorth et al. 2003; Matheson et al. 2003), and the presence of the associated supernova SN2003dh was confirmed later by spectroscopic measurements. Continued monitoring has provided an unprecedentedly rich temporal coverage of the transient in all wavebands (Lipkin et al. 2004; Tiengo et al. 2003; Sheth et al. 2003; Berger et al. 2003; Guziy et al. 2005; Gorosabel et al. 2005a).

We present in this paper the observations done by an Indo-European GRB collaboration at radio, millimeter and optical wavelengths over a total observing span of nearly one year. The millimeter observations were conducted for more than a month at ESO and IRAM. Radio observations at a frequency of $1280 \mathrm{MHz}$ were carried out with the Giant Meter Wave Radio Telescope (GMRT) (http://www.ncra.tifr.res.in) operated by the National Center for Radio Astrophysics, Pune. Optical follow-up was conducted till 34 days after the burst using the $2.01 \mathrm{~m}$ Himalayan Chandra Telescope (HCT) of the Indian Astronomical Observatory (IAO), Hanle and the $1.04 \mathrm{~m}$ Sampurnanand Telescope (ST) at the State Observatory, Naini Tal, now renamed as Aryabhatta Research Institute of Observational Sciences (ARIES). The GMRT observations represent the lowest frequency detection and the longest follow-up of the afterglow reported so far. This is also the first detection of a GRB afterglow by the GMRT. Our optical observations in $U B V R_{\mathrm{C}} I_{\mathrm{C}}$ pass-bands started $\sim 3 \mathrm{~h}$ after the burst. Except in the $R$ band, these represent the earliest photometry of the optical transient. Our optical data also fill many temporal gaps existing in the literature (e.g. Lipkin et al. 2004). At millimeter waves, we used the SEST to make an early detection ( $\sim 0.6$ days) at $86 \mathrm{GHz}$, albeit at low statistical significance, and subsequently monitored the burst until June 19, 2003, using the IRAM Plateau de Bure interferometer, making several simultaneous detections at frequencies ranging from 86 to $240 \mathrm{GHz}$. The early evolution of the optical afterglow followed the familiar behavior of a power-law decay in the light curve, with a steepening ("jet break") around 0.5 days (Garnavich et al. 2003; Smith 2003; Price et al. 2003). The nature of the optical light curve, however, deviated from this simple model after $\sim 1.5$ days, displaying substantial variability and some change in average slope (see Lipkin et al. 2004 for a full compilation). At radio frequencies, the evolution of the afterglow flux was much slower than the early optical decay, and a steepening of the light curve was observed at $\sim 10$ days after the burst (Berger et al. 2003). In order to explain this behavior, Berger et al. (2003) introduced a double-jet model, with a narrow jet responsible for the early optical emission and a wider jet contributing to the radio and late-time optical and X-ray emission.
In addition, the optical light curve also has a significant contribution from the underlying supernova SN2003dh after about a week following the burst. The sharp bump in the optical light curve seen at $\sim 1.5$ days has been attributed by Berger et al. (2003) to the deceleration epoch of the wider jet.

Granot et al. (2003) have instead proposed that the bump in the $R$-band lightcurve at 1.5 days and at three successive epochs could be attributed to refreshed shocks (see also Guziy et al. 2005). In their original model, however, the second jet break at $\sim 10$ days was not expected (Piran et al. 2003).

In this paper, we examine the ability of the double jet model of Berger et al. to fit our observations along with other multi-band observations reported in the literature. We present a refined set of parameters for the two jets resulting from our fits. We also examine whether the two jets are in fact distinct entities or whether a refreshed shock might have converted the decelerating narrow jet to a wider, more energetic jet. In Sects. 2, 3 and 4 we describe respectively our radio, millimeter and optical observations, in Sect. 5 we present theoretical model fits and in Sect. 6 we discuss their implications. In Sect. 7 we calculate the reverse shock emission expected from the model. In Sect. 8 we estimate the possible contribution from SN2003dh. Section 9 summarizes our results.

\section{Radio observations}

We obtained radio observations at the center frequency of $1280 \mathrm{MHz}$ using the Giant Meter Wave Radio Telescope (GMRT) located at Khodad, near Pune in Western India. GMRT is operated by the National Center for Radio Astrophysics. The telescope is an interferometric array of 30 fully steerable parabolic dishes of $45 \mathrm{~m}$ diameter each, spread over a distance of up to $25 \mathrm{~km}$. The telescope operates at several spot frequencies between 150 to $1500 \mathrm{MHz}$, with a maximum bandwidth of $32 \mathrm{MHz}$ at any given band. All feeds provide dual polarization outputs. The highest angular resolution achievable ranges from about 20 arcsec at the lowest frequencies to about $2 \operatorname{arcsec}$ at $1.5 \mathrm{GHz}$. More details about the GMRT can be found in Swarup et al. (1991).

We made the first detection of the GRB 030329 radio transient in $1280 \mathrm{MHz}$ band on 31st March 2003, 2.3 days after the GRB trigger (Rao et al. 2003a). The source was bright, with a flux of $0.33 \mathrm{mJy}, 8 \sigma$ above the background rms noise. Since then we have carried out a series of monitoring observations at the center frequency of $1280 \mathrm{MHz}$, at nine epochs until April 18, 2004. We used a bandwidth of $32 \mathrm{MHz}$ during these observations. The flux scale is set by observing the primary calibrator 3C 286, 3C 147 or 3C 48. A phase calibrator was observed before and after a 30 to 45 min scan on GRB 030329 . The integration time was $16 \mathrm{~s}$. The data recorded from GMRT have been converted to FITS and analyzed using Astronomical Image Processing System (AIPS). At each epoch, the stability of the flux scale was checked by measuring the fluxes of a few background sources. The flux of background sources were consistently stable at all epochs, and the rms fluctuation in their flux (estimated to be $8 \%$ ) has been taken as the error in the flux calibration. The final error quoted is the quadrature sum of the measurement error and the flux calibration uncertainty. The rms 
Table 1. $1280 \mathrm{MHz}$ radio observations at GMRT.

\begin{tabular}{ccc}
\hline \hline & Mid UT & Flux (mJy) \\
\hline 2003 & & \\
& Mar. 31 18:30 & $0.33 \pm 0.09$ \\
& Apr. 1 20:30 & $0.33 \pm 0.09$ \\
& May 30 16:30 & $1.10 \pm 0.15$ \\
& Jun. 28 11:00 & $1.40 \pm 0.16$ \\
& Aug. 9 07:30 & $2.50 \pm 0.25$ \\
& Oct. 20 05:00 & $1.50 \pm 0.17$ \\
& Dec. 3 00:20 & $1.30 \pm 0.14$ \\
2004 & & \\
& Jan. 29 22:12 & $1.20 \pm 0.18$ \\
& Apr. 18 14:32 & $1.10 \pm 0.12$ \\
\hline
\end{tabular}

noise in the image is estimated from a sufficiently large area where no source was visible. The rms noise ranged from 35 to $80 \mu \mathrm{Jy}$ on different epochs. The radio transient was detected in all our observations. The flux showed an initial rise, reaching a peak of $2.5 \mathrm{mJy}$ at $\sim 133$ days after the burst followed by a secular decay (Table 1). As mentioned in Sect. 5, the observed nature of the $1280-\mathrm{MHz}$ light curve tightly constrains the non-relativistic transition of the expanding jet.

\section{The millimeter face of the afterglow}

The first, single dish, observations were carried out on March 29-30, 2003 at La Silla, Chile, with the $15 \mathrm{~m}$ Swedish-ESO Submillimeter Telescope (SEST, Booth et al. 1989). We used the dual channel IRAM SIS-receiver, which allows simultaneous observations at 1.3 and $3 \mathrm{~mm}$ wavelengths. The $1.3 \mathrm{~mm}$ receiver was tuned to $215.0 \mathrm{GHz}$ and the $3 \mathrm{~mm}$ receiver was tuned to $86.243 \mathrm{GHz}$. The backend used was a 3-level correlator with 2000 channels and the bandwidth was $1028 \mathrm{MHz}$ for the $215 \mathrm{GHz}$ receiver, and $512 \mathrm{MHz}$ for the $86 \mathrm{GHz}$ receiver. We used a dual beam switch mode (12 arcmin throw in azimuth) and integrated for $60 \mathrm{~s}$ (on source) per measurement. Observations were performed over three nights (see Table 3). During the observations the sky was clear and stable. The pointing was checked before and after the observations each night and was found to be stable within $\pm 3^{\prime \prime}$.

The intensity scale was calibrated using the standard chopper wheel method, with an internal calibration error of $\sim 10-20 \%$. The intensity scale is converted from K to Jy using $40 \mathrm{Jy} / \mathrm{K}$ and $25 \mathrm{Jy} / \mathrm{K}$ for the $86 \mathrm{GHz}$ and $215 \mathrm{GHz}$ measurements respectively. Two blank sky integrations were done in order to test the performance of the receiver system (see Table 3). These observations were done during day time, resulting in higher noise levels $(16 \pm 164$ and $4 \pm 492 \mathrm{mJy}$ respectively) than the observations of GRB 030329 which were done at night time. The blank sky observations did not produce reliable results at $215 \mathrm{GHz}$.

In order to derive the continuum level, each individual scan was inspected for spikes (which were removed) and abnormal baseline curvature. The average continuum and an estimate of the noise rms were derived for the central $400 \mathrm{MHz}$
Table 3. Results of the ESO/SEST scans on GRB 030329.

\begin{tabular}{lccc}
\hline \hline UT date of 2003 & Freq. (GHz) & flux (mJy) & Beam \\
\hline Mar. 29/30 & 86 & $82 \pm 52$ & $57^{\prime \prime}$ \\
& 215 & $201 \pm 308$ & $23^{\prime \prime}$ \\
Mar. 30/31 & 86 & $-2 \pm 78$ & $57^{\prime \prime}$ \\
& 215 & $-49 \pm 262$ & $23^{\prime \prime}$ \\
Mar. 31/Apr. 1 & 86 & $38 \pm 65$ & $57^{\prime \prime}$ \\
& 215 & $-144 \pm 250$ & $23^{\prime \prime}$ \\
\hline
\end{tabular}

of the $86 \mathrm{GHz}$ spectra, and $700 \mathrm{MHz}$ of the $215 \mathrm{GHz}$ spectra. The subscans were subsequently added together, weighted with their individual noise rms. Scans deviating by more than 3 sigma from this initial analysis were removed and the procedure repeated with the remaining scans. This generally does not change the average continuum flux, but decreases the dispersion. The final average continuum fluxes are given in Table 4. The dispersion in the continuum fluxes among individual scans are given as the $1 \sigma$ error in Table 4.

The dispersion in the measured continuum level, using the present technique, reflects the variable sky background and the inherent calibration uncertainty. It does not follow a normal distribution and increasing the number of subscans does not necessarily decrease the dispersion. The dispersion of continuum fluxes follows more a top-hat distribution. As such, it is not a good measure of the precision of the final continuum flux value, but nevertheless the only measure of the uncertainty at our disposal.

Under clear and stable weather conditions, as was the case for the SEST observations of GRB 030329, the average flux value is usually a robust estimate if the number of subscans is sufficiently large (e.g. >20). The flux value derived for the night of March 29 is higher than any of the following measurements, while the noise rms remains more or less constant. This is a strong indication that continuum flux was detected during the first night but not during the following nights. The weather conditions at the higher frequency band $(215 \mathrm{GHz})$ were worse than at $86 \mathrm{GHz}$. We therefore conclude that at least at $3 \mathrm{~mm}$ $(86 \mathrm{GHz})$, we did tentatively detect continuum flux during March 29, at a level around $80 \mathrm{mJy}$. The uncertainty associated with this, however, remains poorly defined and the quoted flux value should be regarded as approximate. At a $2 \sigma$ level, the quoted uncertainty gives an upper bound of $\sim 104 \mathrm{mJy}$ on the flux of the source.

At Plateau de Bure interferometer (Guilloteau et al. 1992), we carried out simultaneous observations in two bands, around $90 \mathrm{GHz}$ and $230 \mathrm{GHz}$, the actual band center frequencies being slightly different on different days. The instrument was operating on compact D configuration with six antennas (6Dp) until May 3rd and with fewer antennas later (see Table 4). Data reduction and analysis was performed with the GLIDAS (Grenoble Image and Line Data Analysis) software. The target was observed at coordinates RA (2000) = $10^{\mathrm{h}} 44^{\mathrm{m}} 50.030^{\mathrm{s}}$, Dec $(2000)=+21^{\circ} 31^{\prime} 18.15^{\prime \prime}$ and the best position offsets were found on 31 Mar. 2003 to be: $-0.87^{\prime \prime} \pm$ $0.03^{\prime \prime}$ and $-0.78^{\prime \prime} \pm 0.02^{\prime \prime}$ respectively. Therefore, the absolute 
Table 4. Results of the IRAM/PdB scans on GRB 030329.

\begin{tabular}{|c|c|c|c|c|}
\hline UT date of 2003 & Config. & $\begin{array}{c}\text { freq } \\
(\mathrm{GHz})\end{array}$ & $\begin{array}{c}\text { flux } \\
\text { (mJy) }\end{array}$ & Beam and PA \\
\hline \multirow[t]{2}{*}{31 Mar. 00:04 to 03:07 } & $6 \mathrm{Dp}$ & 86.253 & $58.6 \pm 0.5$ & $14.1^{\prime \prime} \times 4.0^{\prime \prime}$ at $55^{\circ}$ \\
\hline & & 232.032 & $46.8 \pm 3.1$ & $5.2^{\prime \prime} \times 1.5^{\prime \prime}$ at $55^{\circ}$ \\
\hline \multirow[t]{2}{*}{31 Mar. $18: 33$ to $23: 57$} & $6 \mathrm{Dp}$ & 98.473 & $58.2 \pm 0.6$ & $6.4^{\prime \prime} \times 4.2^{\prime \prime}$ at $98^{\circ}$ \\
\hline & & 238.500 & $40.6 \pm 2.1$ & $2.8^{\prime \prime} \times 1.6^{\prime \prime}$ at $84^{\circ}$ \\
\hline \multirow[t]{2}{*}{1 Apr. $20: 20$ to $21: 55$} & $6 \mathrm{Dp}$ & 86.673 & $51.7 \pm 0.9$ & $9.1^{\prime \prime} \times 4.2^{\prime \prime}$ at $98^{\circ}$ \\
\hline & & 240.528 & $23.8 \pm 3.2$ & $3.9^{\prime \prime} \times 1.6^{\prime \prime}$ at $100^{\circ}$ \\
\hline 5 Apr. $16: 54$ to $18: 04$ & $6 \mathrm{Dp}$ & 115.447 & $40.4 \pm 3.7$ & $10.3^{\prime \prime} \times 3.1^{\prime \prime}$ at $-53^{\circ}$ \\
\hline \multirow[t]{2}{*}{10 Apr. $17: 42$ to $19: 12$} & $6 \mathrm{Dp}$ & 86.244 & $23.5 \pm 0.4$ & $11.0^{\prime \prime} \times 4.2^{\prime \prime}$ at $-62^{\circ}$ \\
\hline & & 232.171 & $12.8 \pm 1.3$ & $4.5^{\prime \prime} \times 1.5^{\prime \prime}$ at $-65^{\circ}$ \\
\hline \multirow[t]{2}{*}{14 Apr. $20: 10$ to $22: 16$} & $6 \mathrm{Dp}$ & 91.333 & $14.9 \pm 0.4$ & $8.1^{\prime \prime} \times 4.2^{\prime \prime}$ at $84^{\circ}$ \\
\hline & & 217.029 & $9.2 \pm 2.2$ & $3.6^{\prime \prime} \times 1.7^{\prime \prime}$ at $80^{\circ}$ \\
\hline \multirow[t]{2}{*}{18 Apr. $19: 39$ to $21: 10$} & $6 \mathrm{Dp}$ & 115.271 & $7.7 \pm 1.0$ & $6.3^{\prime \prime} \times 3.3^{\prime \prime}$ at $90^{\circ}$ \\
\hline & & 232.032 & $6.5 \pm 1.8$ & $3.3^{\prime \prime} \times 1.5^{\prime \prime}$ at $87^{\circ}$ \\
\hline \multirow[t]{2}{*}{24 Apr. $17: 36$ to $19: 32$} & $6 \mathrm{Dp}$ & 96.250 & $4.7 \pm 0.7$ & $8.2^{\prime \prime} \times 4.0^{\prime \prime}$ at $-67^{\circ}$ \\
\hline & & 241.480 & $0.0 \pm 3.8$ & $3.5^{\prime \prime} \times 1.4^{\prime \prime}$ at $105^{\circ}$ \\
\hline \multirow[t]{2}{*}{3 May $15: 59$ to $19: 49$} & $6 \mathrm{Dp}$ & 86.243 & $2.9 \pm 0.3$ & $9.5^{\prime \prime} \times 4.4^{\prime \prime}$ at $108^{\circ}$ \\
\hline & & 233.467 & $1.4 \pm 1.3$ & $3.6^{\prime \prime} \times 1.6^{\prime \prime}$ at $-76^{\circ}$ \\
\hline \multirow[t]{2}{*}{16 May $13: 20$ to $15: 57$} & $5 \mathrm{Dp}$ & 86.243 & $1.1 \pm 0.8$ & $12.4^{\prime \prime} \times 5.3^{\prime \prime}$ at $-54^{\circ}$ \\
\hline & & 231.490 & $0.0 \pm 10.1$ & $4.6^{\prime \prime} \times 1.9^{\prime \prime}$ at $-59^{\circ}$ \\
\hline \multirow[t]{2}{*}{28 May $21: 29$ to $23: 22$} & $5 \mathrm{Dp}$ & 84.443 & $0.4 \pm 0.7$ & $16.6^{\prime \prime} \times 5.5^{\prime \prime}$ at $52^{\circ}$ \\
\hline & & 238.500 & $0.0 \pm 8.0$ & $5.5^{\prime \prime} \times 1.9^{\prime \prime}$ at $52^{\circ}$ \\
\hline 20 June $12: 41$ to $17: 49$ & $4 \mathrm{Dp}$ & 95.434 & $0.6 \pm 1.3$ & $5.92^{\prime \prime} \times 5.63^{\prime \prime}$ at $64^{\circ}$ \\
\hline
\end{tabular}

coordinates are RA $(2000)=10^{\mathrm{h}} 44^{\mathrm{m}} 49.968^{\mathrm{s}}( \pm 0.002 \mathrm{~s})$, Dec $(2000)=+21^{\circ} 31^{\prime} 17^{\prime \prime} 37\left( \pm 0.02^{\prime \prime}\right)$. Thus, within the error limits, the measured position definitely stays constant throughout the observing period. The source has been detected up to May 3rd, after which there are only upper limits available.

\section{Optical observations and data reduction}

Starting about $3 \mathrm{~h}$ after the burst, we obtained a total of 13, 70, 87, 167, and 39 photometric observations in Johnson $U$, $B, V$ and Cousins $R$ and $I$ bands respectively, from both ST and HCT. The CCD used at HCT was $1024 \times 1024$ pixel $^{2}$ with the entire chip covering a field of $\sim 4.7 \times 4$ '. $^{\prime}$ on the sky. It has a read out noise of $11 \mathrm{e}^{-}$and gain of $4.8 \mathrm{e}^{-} / \mathrm{ADU}$. A CCD chip of size $2048 \times 2048$ pixel $^{2}$ was used at ST, which covers a field of $\sim 13^{\prime} \times 13^{\prime}$. The gain and read out noise are $10 \mathrm{e}^{-} / \mathrm{ADU}$ and $5.3 \mathrm{e}^{-}$respectively. The frames were binned in $2 \times 2$ pixel $^{2}$ to improve the signal-to-noise ratio of the source. Several twilight flat field and bias frames were obtained for the CCD images at both the telescopes. While imaging the optical transient (OT), several short exposures upto a maximum of $15 \mathrm{~min}$ were taken in various filters. We used MIDAS, IRAF and DAOPHOT softwares to process the CCD frames in the standard fashion. The bias subtracted and flat fielded CCD frames were co-added, whenever found necessary.

The BVRI magnitudes of the OT obtained from the Sampurnanand Telescope at Naini Tal were calibrated differentially using secondary standard stars Nos. 1, 11, 14, 19, 37 and 57 in the list of Henden (2003), while the $U$ magnitudes were determined using reference stars 1,11 and 37 from Henden (2003). BVR magnitudes obtained at the IAO were also calibrated differentially, using reference stars Nos. 14, 18 and 19 of Henden (2003). OT magnitudes at similar epochs, determined using photometry from the two sites, are consistent with each other within the limits of uncertainty. A full compilation of our data is presented in Table 2.

Figure 1a displays our $U B V R I$ photometry (open circles) along with some of the data published by other authors (filled circles). As mentioned above, in $U B V I$ bands we report the earliest observations and in all the bands our observations fill in gaps in photometric data reported in the literature so far, at epochs beyond one day after the burst.

Reported X-ray observations of this afterglow are shown in an accompanying Fig. 1b. The role of the X-ray observations in constraining the model, along with observations in other bands, is discussed in Sect. 5.

\section{Modeling the multiband observations}

It was proposed by Rhoads (1999) that the explosion which makes the GRB may not be isotropic. Ejection of matter in the explosion could in fact be collimated within narrow cones. If so, then a signature of this collimation is expected in the form of an achromatic steepening ("jet break") in the afterglow light 


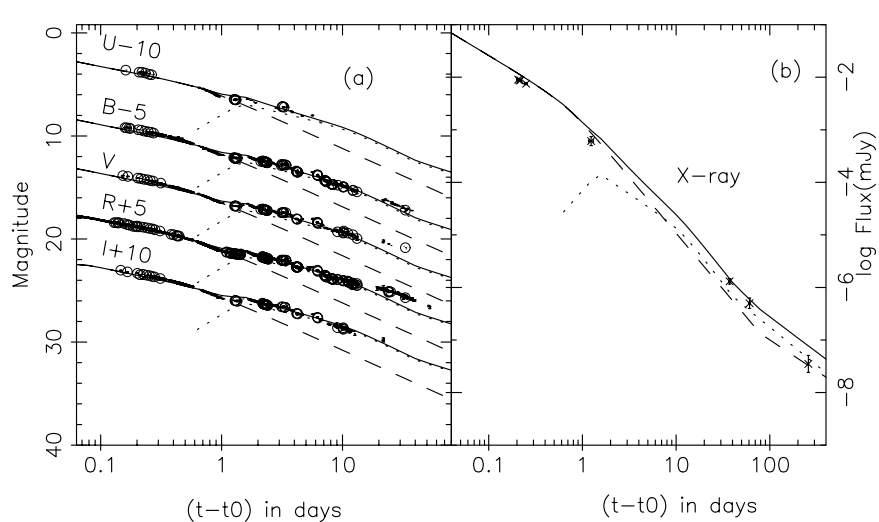

Fig. 1. a) The optical lightcurve of the afterglow of GRB 030329. Open circles represent the data presented in this paper and filled circles are those from the literature (Lipkin et al. 2004). The solid line shows the total flux predicted by model 1 discussed in the text, which has two jets as in Berger et al. (2003). The dashed line shows the contribution of the narrow jet alone and the dotted line that of the wide jet in each band. b) X-ray observations reported by Tiengo et al. (2003, 2004), shown along with the prediction of model 1. Contribution of the narrow jet and the wide jet are shown separately as the dashed and the dotted line respectively. The total flux is shown as the solid curve.

curve (Rhoads 1999; Sari et al. 1999). The jet break appears when the Lorentz factor of the expanding outflow drops below the inverse of the initial angle of collimation, causing the lateral expansion of the jet to dominate over its radial motion. This behavior has now been observed in a number of afterglows (see reviews by Piran 2004 and Sagar 2002).

\subsection{The double jet model for GRB 030329}

The X-ray and optical lightcurves of GRB 030329 afterglow had an initial temporal slope of $\sim-0.9$. Around half a day, the optical decay steepened to an index of $\sim-1.9$. Sampling of the $\mathrm{X}$-ray evolution was poor, but an interpolation of the $R X T E$ and $X M M$ data obtained during $\sim 0.1$ to $\sim 100$ days indicated a break almost at the same time as the optical steepening mentioned above (Tiengo et al. 2003). A nearly simultaneous break observed in frequencies separated by four orders of magnitude suggested that the break observed at $\sim 0.5$ days was a jet break.

At radio frequencies, the first observations obtained were around 1 days, somewhat later than the epoch of the jet break obtained from optical and X-ray observations. The radio and millimeter light curves, however, did not display the behavior expected after a jet break at 0.5 days. Instead, they were rather well described by a second jet, with a jet break around 10 days (Berger et al. 2003; Sheth et al. 2003). The optical light curve showed a re-brightening around 1.5 days, followed by a slower decay consistent with that expected from the second jet to which the radio emission was attributed (Berger et al. 2003; Lipkin et al. 2004). This led Berger et al. (2003) to propose a double-jet model for GRB 030329. The optical re-brightening at 1.5 days was attributed to the epoch of deceleration of the second jet. Berger et al. (2003) estimated the initial opening angle of the first (narrow) jet to be $\sim 5^{\circ}$ and that of the second (wide) jet to be $\sim 18.4^{\circ}$. The energy contents of the two jets were estimated to be $\sim 6.7 \times 10^{48}$ erg for the narrow jet and $\sim 10^{49}$ erg for the wide jet. Compared to the narrow jet, a later deceleration epoch implied a smaller initial Lorentz factor for the wide jet, and correspondingly a much higher initial baryon load. The double jet model has been found to be consistent with most observations reported till date. The optical emission at late times ( $t>10$ days) is, however, dominated by the associated supernova SN2003dh.

We attempted modeling our observations along with multiwavelength data available in the literature within the ambit of this double-jet model (model 1). The basic quantity in our model is the synchrotron source function, which we consider to have appropriate power-law forms between the usual break frequencies. Transition from one power-law phase to another is made gradual through a Band type smoothening (Band et al. 1993) at the peak frequency $v_{\mathrm{m}}$ and the cooling frequency $v_{\mathrm{c}}$. The self-absorption frequency $v_{\mathrm{a}}$ is not treated as a break; instead the absorption is incorporated into the expression for synchrotron optical depth, which, along with the source function, yields the flux at any given frequency. In the co-moving frame of the shock, the optical depth is set to unity at $v=v_{\mathrm{a}}$ (comoving). We incorporate transition to a non-relativistic phase of expansion, as in Frail et al. (2000). The non-relativistic transition is treated as a sharp break at a time $t=t_{\mathrm{nr}}$. We obtain our fits through the usual $\chi^{2}$ minimization procedure, using $v_{\mathrm{a}}, v_{\mathrm{m}}, v_{\mathrm{c}}, t_{\mathrm{nr}}$, the electron distribution index $p$ and the jet break time $t_{\mathrm{j}}$ as the fit parameters. Here we are trying to model the underlying smooth power law behavior rather than the short time scale variabilities in the lightcurve. Since our model does not include the short-term variabilities, the nominal $\chi^{2}$ obtained is relatively high. The best fit from this model is shown in Figs. 1 and 2. We derived the physical parameters $E_{\text {iso }}$ (the isotropic equivalent energy of the burst), $n$ (number density of the ambient medium), $\epsilon_{\mathrm{e}}$ and $\epsilon_{\mathrm{B}}$ (fractional energy content in the electrons and in the magnetic field respectively) using the expressions in Wijers \& Galama (1999) from these fitted parameters, with appropriate modifications to place $v_{\mathrm{a}}$ at $\tau_{v}=1$ instead of 0.35 used by Wijers \& Galama (1999).

\subsubsection{The wide jet}

The parameters of the wide jet are well constrained by data in the 4-250 GHz range, as discussed by Berger et al. (2003) and Sheth et al. (2003). At the jet break time of 9.8 days, we find the self absorption frequency $v_{\mathrm{a}}$ to be $1.3_{-0.06}^{+0.25} \times 10^{10} \mathrm{~Hz}$, the synchrotron peak frequency $v_{\mathrm{m}}$ to be $3.98_{-0.1}^{+0.5} \times 10^{10} \mathrm{~Hz}$ with a peak flux $F_{\mathrm{m}}$ of $44.7_{-2.0}^{+1.0} \mathrm{mJy}$. The post jet-break decay in radio, the peak optical flux at 1.5 days and the late X-ray observations at 37 and 61 days together constrain the electron energy distribution index $p$ to $2.3_{-0.02}^{+0.05}$ and the cooling frequency $v_{\mathrm{c}}$ to $3.98_{-2.0}^{+1.3} \times 10^{14} \mathrm{~Hz}$ after the jet break. These parameter values are very similar to those derived by Berger et al. (2003). Our $1280 \mathrm{MHz}$ observations show a gradual rise of flux to a peak around 133 days. This behavior can be reproduced by a nonrelativistic transition of the jet at $t_{\mathrm{nr}}=42_{-7}^{+17}$ days. 


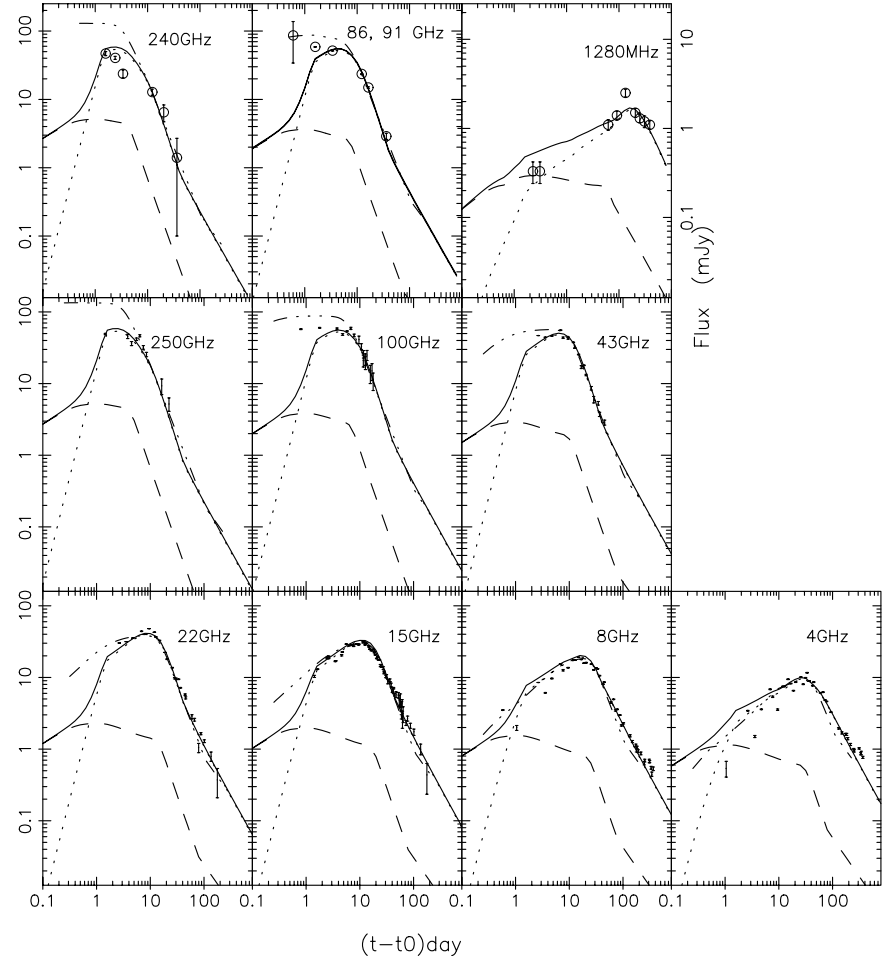

Fig. 2. Millimeter and radio observations of GRB 030329 afterglow along with the predictions of model 1 (two jets). Observations reported in this paper are represented by open circles, crosses are data points from Berger et al. (2003) and Sheth et al. (2003). The dashed and the dotted lines represent contributions of the narrow and the wide jet respectively, the solid line shows the sum. The dash-dot-dot line shows a model fit for an assumed stellar-wind density profile for the ambient medium.

\subsubsection{The narrow jet}

The jet break time for the narrow jet is derived to be $0.69_{-0.06}^{+0.08}$ days from the optical light curve. Using a galactic extinction $E(B-V)=0.025 \mathrm{mag}$ in the direction of the GRB (Schlegel et al. 1998), the early optical and X-ray observations, both before and after this break, are well described by an electron energy distribution index $p$ of $2.12 \pm 0.05$. Some authors (e.g. Sato et al. 2003; Lipkin et al. 2004) have conjectured that there occurs a passage of the cooling break $\left(v_{\mathrm{c}}\right)$ through optical bands within the first few hours after the GRB, based on a derived change in slope of $R$-band light curve around $\sim 0.25$ days, and a small color evolution. Our own observations have a continuous coverage from $\sim 0.15$ days to $\sim 0.3$ days after the burst, taken from the same instrument and calibrated uniformly on the same scale. We find that our data over this interval are fit very well by a single power law. Even the data reported by Lipkin et al. (2004) and Sato et al. (2003) do not conclusively demonstrate a secular steepening at $\sim 0.25$ days; the effect could be easily mimicked by short-term variability riding on a single, underlying power law. Colors derived from our own multiband observations have somewhat large errors $(\sim 0.08 \mathrm{mag} 1-\sigma)$, and we are unable to discern the $\sim 0.1$ mag systematic change in $B-R$ color reported by Lipkin et al. (2004). As mentioned by Lipkin et al. (2004), there could be various reasons for this early color evolution. We do not feel that the passage of cooling break through optical bands at early times can be conclusively established from the existing observations. From multiband fits, however, we estimate $v_{\mathrm{c}}$ to be at $1.0_{-0.5}^{+1.0} \times 10^{16} \mathrm{~Hz}$ at 0.5 days. At times $>1.5$ days, the contribution from the wide jet is sufficient to reproduce the radio light curves (see Fig. 2), so the radio emission from the narrow jet is constrained to be almost negligible. In order to achieve this, we need to have the peak frequency $v_{\mathrm{m}}$ and the self absorption frequency $v_{\mathrm{a}}$ of the narrow jet to be as high as possible. The passage of $v_{\mathrm{m}}$ is not observed through the optical band, so we chose it to be just below the $R$-band at the earliest epoch $(\sim 0.05$ days $)$ at which data are available. This results in $v_{\mathrm{m}}$ regressing to $\sim 10^{13} \mathrm{~Hz}$ at the jet break epoch of 0.5 days. The fitted peak flux $F_{\left(v=v_{m}\right)}$ is $19.8_{-2.4}^{+9.3} \mathrm{mJy}$ at this time. The density of the ambient medium can be derived from the rather well-constrained parameters of the wide jet, and works out to be $n \sim 8$ atom/cc. This, along with the narrow jet parameters mentioned above, predicts the self absorption frequency of the narrow jet to be $v_{\mathrm{a}}$ equal to $3.1_{-0.63}^{+0.14} \times 10^{9} \mathrm{~Hz}$ at 0.5 days. We find that this value of $v_{\mathrm{a}}$ yields adequate suppression of the narrow jet flux at low frequencies for the model to be consistent with our $1280 \mathrm{MHz}$ data.

As mentioned earlier, the $\chi_{\text {d.o.f. }}^{2}$ is somewhat high due to short-term variabilities in the observed light curve. From the fit we excluded the first seven days of data at 4.86 and $8.46 \mathrm{GHz}$, which appear to have been affected by scintillations. The first data point at $250 \mathrm{GHz}(\sim 1.5$ days $)$ and at $100 \mathrm{GHz}$ (0.8 days) were also removed from the fit. We did not consider five more data points in radio bands (of 2.6 days in $43 \mathrm{GHz}$, days 1 and 12 in $22 \mathrm{GHz}$ and days 3.5 and 4.7 in $15 \mathrm{GHz}$ ) which produced high $\chi^{2}$ values due to scatter. We exclude the optical data from discussion for epochs larger than $\sim 5$ days because of the dominant contribution from SN2003dh. A $\chi_{\text {d.o.f. }}^{2}$ of 23.3 is obtained for the best fit with this model. The optical (mostly $V$ and $B$ ) bands dominate the contribution to $\chi^{2}$ along with the lower radio frequencies ( $4 \mathrm{GHz}, 8 \mathrm{GHz}$ and $15 \mathrm{GHz}$ ). The number density of the ambient medium is inferred to be $8.6_{-5}^{+12}$. We infer the fractional energy content in relativistic electrons and magnetic field to be $0.56_{-0.5}^{+0.4}$ and $4_{-1.8}^{+1.9} \times 10^{-4}$ respectively for the narrow jet, and $9.0_{-1}^{+3} \times 10^{-2}$ and $11.9_{-7}^{+10} \times 10^{-4}$ for the wide jet. We derive $1.4_{-0.8}^{+1.3} \times 10^{51} \mathrm{erg}$ for the isotropic equivalent energy and $6.2_{-0.03}^{+0.02}$ degrees for the opening angle of narrow jet. This corresponds to a total energy content of $3.3_{-2.4}^{+4.8} \times 10^{48} \mathrm{erg}$ in the jet. For the wide jet, we derive an isotopic equivalent energy of $1.2_{-0.2}^{+0.4} \times 10^{50} \mathrm{erg}$, opening angle of $23.3_{-0.04}^{+0.07}$ degrees and a total energy of $5.0_{-2.1}^{+3.3} \times 10^{48} \mathrm{erg}$.

We calculated the rising flux from the wide jet at $t<$ 1.5 days, assuming time evolution of the spectral parameters to be of the form $v_{\mathrm{m}} \propto t^{0}, v_{\mathrm{c}} \propto t^{-2}, f_{v_{\mathrm{m}}} \propto t^{3}$ (Peng et al. 2004) and $v_{\mathrm{a}} \propto t^{1}$ and normalizing them at 1.5 days.

We also explored the possibility of the ambient medium of the burst being generated by a stellar wind, with a density profile of $n(r) \propto r^{-2}$, but were unable to obtain consistent fits with the double-jet model. If the model is tuned to reproduce radio data in the $8-43 \mathrm{GHz}$ frequency range, it leads to an overprediction of fluxes in millimeter bands and an underprediction at $1280 \mathrm{MHz}$ (cf. Fig. 2). 


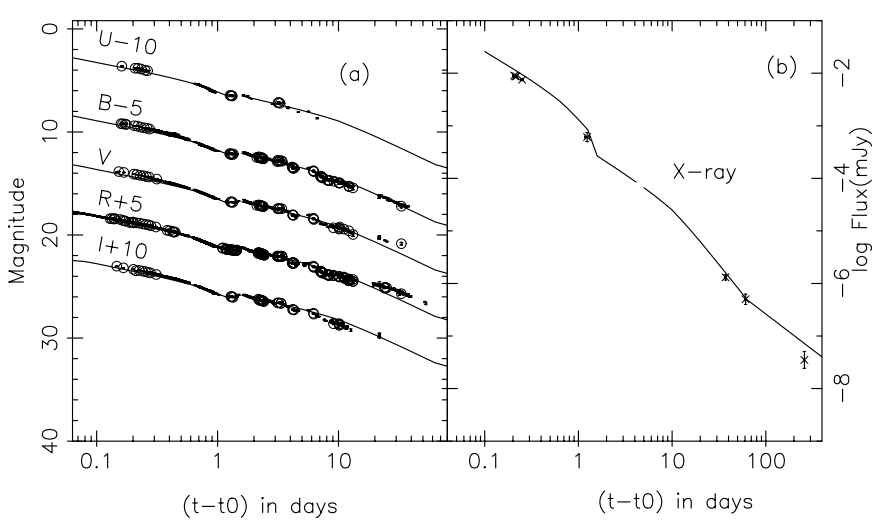

Fig. 3. a) The optical lightcurve of the afterglow of GRB 030329, shown with the prediction of model 2 (solid line), which assumes a transition of an initially narrow jet to a wider jet at $\sim 1.5$ days. b) X-ray observations reported by Tiengo et al. (2003, 2004), with predictions of model 2. The flattening seen at late times is due to the transition into non-relativistic regime at $\sim 63$ days.

\subsection{Refreshed jet?}

Most of the observations are well reproduced by a model which sums the contributions from the wide and narrow jet. We note that the contribution of the narrow jet is almost negligible at radio bands; in fact the wide jet alone is quite sufficient to account for the observed flux after $\sim 1.5$ days. It therefore appears to us that the data could be well described if, instead of both jets contributing simultaneously to the emission, the narrow jet alone contributes at epochs earlier than $\sim 1.5$ days, and only the wide jet after that time. This suggests that a possible reenergization event around or before $\sim 1.5$ days could have refreshed the initially narrow jet, which had entered the lateral expansion phase, and given it additional forward momentum, converting it into the second, "wide" jet. The opening angle of the laterally expanding, initially narrow jet around $\sim 1.5$ days is estimated to be $\sim 20^{\circ}$, not far from the initial opening angle inferred for the wide jet $\left(\sim 23^{\circ}\right)$. We therefore consider it possible that a re-energization event occurred after the jet break of the narrow jet as suggested by Granot et al. (2003) and the doublejet model for GRB 030329 could represent the conversion of an initially narrow jet to a wide one by the re-energization.

In a simple representation of such a re-energization, we assume that the physical parameters of the fireball, namely $E, \epsilon_{\mathrm{e}}$ and $\epsilon_{\mathrm{B}}$ undergo a change after re-energization, while the ambient density $n$ remains the same. We allow these physical parameters to be determined by the model fits.

Fits to multiwavelength observations with this model (model 2) are shown in Figs. 3 and 4 . We excluded the same set of data in these fits as done in model 1. The minimum $\chi_{\text {d.o.f. }}^{2}$ we obtained with this model is 24.5 , slightly higher than the value for the previous model. Here again, the $\chi^{2}$ is dominated by the optical band as well as the low radio frequencies. Both model 1 and model 2 underpredict the flux at $1280 \mathrm{MHz}$ peak by a factor of 2 . But it is difficult to make a distinction between model 1 and model 2 from the small difference in the $\chi^{2}$. Parameters for the initial, narrow jet are nearly the same as those listed in Sect. 5.1.2. except

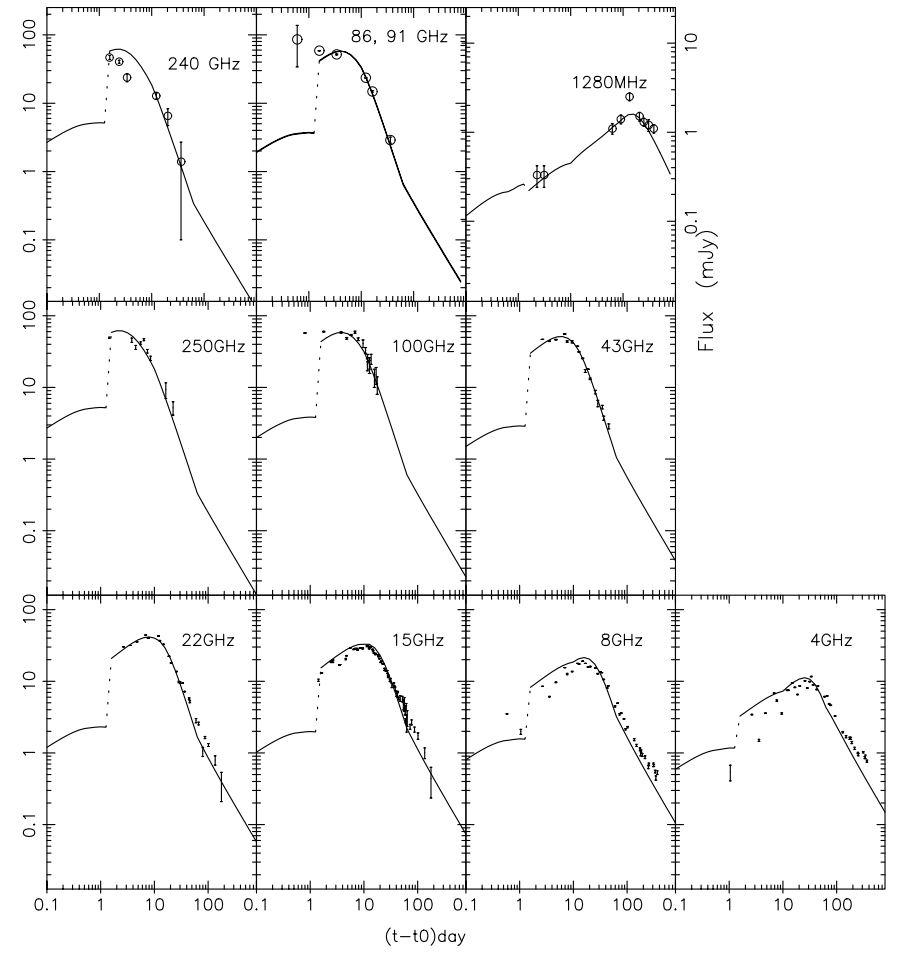

Fig. 4. Millimeter and radio observations of GRB 030329, along with the predictions of model 2 (refreshed jet) shown as the solid line. Open circles are data presented in this paper, crosses represent data taken from Berger et al. (2003) and Sheth et al. (2003).

the self absorption frequency $v_{\mathrm{a}}\left(2.9_{-0.06}^{+0.8} \times 10^{9} \mathrm{~Hz}\right)$, which is implied by the value of $n$ inferred from the parameters of the jet after re-energization. Parameters of the refreshed jet are only marginally different from that of the wide jet discussed in Sect. 5.1.1. We obtain a $p$ of $2.24 \pm 0.02$, $t_{\mathrm{j}}$ of $10_{-1.0}^{+2.3}$ days, and at 9.8 days, a cooling frequency of $5.0_{-1.5}^{+2.1} \times 10^{14} \mathrm{~Hz}$, and a marginally reduced self absorption frequency of $1.1_{-0.05}^{+0.3} \times 10^{10} \mathrm{~Hz}$. The non-relativistic transition time $t_{\mathrm{nr}}$ is $63_{-30}^{+13.5}$ days. The number density of the ambient medium is inferred to be $6.7_{-3}^{+13}$. We infer the fractional energy content in relativistic electrons $\left(\epsilon_{\mathrm{e}}\right)$ and magnetic field $\left(\epsilon_{\mathrm{B}}\right)$ to be $0.53_{-0.4}^{+0.5}$ and $4_{-1.3}^{+2.5} \times 10^{-4}$ respectively for the initial (narrow) jet, and $0.103_{-0.01}^{+0.05}$ and $1.0_{-0.5}^{+1} \times 10^{-3}$ for the refreshed (wide) jet. We derive an isotropic equivalent energy of $1.2_{-0.6}^{+1.6} \times 10^{51}$ erg for the original jet, which has an initial opening angle of $5.9_{-0.1}^{+0.3}$ degrees. This corresponds to a total energy content of $3.2_{-2.8}^{+6} \times 10^{48} \mathrm{ergs}$ in the jet. After re-energization, which is assumed to end around $\sim 1.5$ days, the total energy content of the jet increases to $5.8_{-1.7}^{+5.9} \times 10^{48} \mathrm{erg}$, and the jet widens to $20.5_{-0.03}^{+0.1}$ degrees.

The transition to the refreshed physical parameters of the jet is expected to be gradual, over the time required to establish a new equilibrium. The timescale for achieving a new BlandfordMcKee structure will be roughly equal to the time the second wave requires to cross the existing shocked shell. This time, in the co-moving frame, can be estimated as the thickness of the matter in the co-moving frame of the shock, divided by $c$, which in the observer's frame will be $\Delta t \approx R /\left(a \Gamma_{\text {old }} \Gamma_{\text {new }} c\right)$, where $a \sim 5-10$. At 1.5 days, when the new power law 
phase begins, we calculate the bulk Lorentz factor $\left(\Gamma_{\text {new }}\right)$ to be $\sim 2.3$, by extrapolating the value of $\Gamma$ from the jet break time. Extrapolation for the initial jet produces $\Gamma_{\text {old }}$ to be close to this value. Hence $\Delta t$ will be of the order of $0.2-0.3$ days. From a close examination of the optical lightcurve, we find that the refreshment episode could begin at $\sim 1.2$ days and at $\sim 1.5$ days, the new power law phase begins.

\section{Discussion}

We have seen above a comparison between the parameters of the two models. We now discuss their compatibility with the constraints imposed by other available observations of the afterglow. The angular size of the fireball estimated by Taylor et al. (2004) can be reproduced by models with isotropic equivalent energy to external density ratio in the range $10^{50}-10^{52} \mathrm{erg} \mathrm{cm}^{-3}$ (Oren et al. 2004). The parameters extracted from both the models fall close to this range.

Polarization measurements of the afterglow are available in optical (Greiner et al. 2003b) and in radio (Taylor et al. 2004) bands. In the optical, the degree of polarization decreases shortly after the jet break at $\sim 0.5$ days and rapid variations in polarization start occurring around 1.5 days, which according to Greiner et al. could be the beginning of a new power law phase. The linear polarization at $8 \mathrm{GHz}(<0.1 \%)$ around 8 days is significantly lower than the optical polarization $(\sim 2 \%)$, which could be due to the fireball being optically thick at this frequency. This polarization behavior has been thought to support the double jet model (Greiner et al. 2003b; Taylor et al. 2004); however this is equally applicable to the refreshed jet.

We point out that the first millimeter-wave observation at $86 \mathrm{GHz}$ by the SEST at 0.6 days and the $100 \mathrm{GHz}$ observation reported by Sheth et al. (2003) at 0.8 days are not well fit by this simple model. Nor does the double-jet model of Berger et al. (2003) succeed in reproducing this well.

\section{Reverse shock}

It has been pointed out (Piran et al. 2003) that the deceleration of the wide jet at 1.5 days is expected to be accompanied by a strong radio flash from the reverse shock, which is not observed. This concern remains for the refreshed jet model, too. The two models differ in the nature of the medium in which the second shock front decelerates (in model 1, a normal ISM while in model 2, it is the material already shocked by the first shell). The Sedov length in these two cases are also to be evaluated differently, since in model 1 , the shell responsible for the wide jet encounters matter all the way from the progenitor star while in model 2, the second wave of energy passes through the region evacuated by the first jet.

We estimated the reverse shock emission expected in either model, assuming that the shock is ultrarelativistic (thick shell case) (Sari \& Piran 1995; Kobayashi 2000). For model 1 there are four relevant regions, namely, normal ISM (0), ISM shocked by the wide jet (1), reverse shocked ejecta (2) and the cold second shell (3). Region 1 and region 2 are separated by a contact discontinuity (CD). We followed the formulation of Kobayashi (2000) to obtain the flux expected from reverse shock. In model 2, since the reverse shock originates when the second wave of energy decelerates into the already shocked material, the space ahead of the CD will be divided into three regions instead of two (Kumar \& Piran 2000). The five relevant regions in this case are: the ISM (0), the ISM shocked by the first wave (1), ISM additionally shocked by the second wave (2), reverse shocked ejecta (3) and the cold second shell (4). A contact discontinuity separates regions 2 and 3. Assuming pressure balance at the $\mathrm{CD}$, and for the ejecta using the assumption that $n_{4} R^{2}$ is constant, (where $n_{4}$ is the number density of the cold shell and $R$ is the distance to the $\mathrm{CD}$ ), one obtains the bulk Lorentz factor $\left(\gamma_{3}\right)$ and thermodynamic quantities (density and pressure) of region 3 . These quantities allow one to estimate the synchrotron emission from that region. The thickness $(\Delta)$ of the ejecta is an unknown parameter in both models. For a given $\Delta$ the peak flux produced by model 1 is two orders of magnitude lower than model 2 at the deceleration time of 1.5 days. The computed flux is inversely related to $\Delta$, and the minimum value $\Delta$ can reach without overpredicting the flux observed at various bands is $\sim 10^{10} \mathrm{~cm}$ for model 1 , and $\sim 10^{13} \mathrm{~cm}$ for model 2 . More detailed investigations taking into account detailed hydrodynamics in non-spherical geometry as well as the density structure of a post-jet break fireball may be necessary to get a better estimate.

\section{SN2003dh}

The optical emission observed at times later than $\sim 7$ days cannot be fully accounted for by the afterglow models discussed so far. We attribute the excess emission at these late epochs primarily to the associated supernova, SN2003dh. We subtract the afterglow flux predicted by the model and the flux due to the host galaxy (Gorosabel et al. 2005b) from the observed data to estimate the contribution from the supernova. Figure 5 displays the flux attributed to the supernova in the two models discussed above. The K-corrected light curve of SN1998bw (Galama et al. 1998) appropriate for the redshift of GRB 030329 is shown along with the residuals for comparison. While being similar in temporal behavior, the residuals from model 1 are fainter by $\sim 0.3$ mag in comparison to an equivalent SN1998bw lightcurve. In case of model 2 , this difference is $\sim 0.4$ mag. These results compare well with the estimate of Lipkin et al. who find that an SN1998bw lightcurve, diminished by $0.3 \mathrm{mag}$, is required to fit the observed data.

\section{Summary}

We have presented low frequency radio, millimeter wave and optical observations of the afterglow of GRB 030329, and interpreted them in terms of the double-jet model discussed earlier in the literature. Our main conclusions are summarized below.

- The $1280 \mathrm{MHz}$ GMRT observations, starting 2 days after the burst and continuing for over a year, provide constraints on the self absorption frequency of the emission region as well as the epoch of non-relativistic transition of the fireball. 


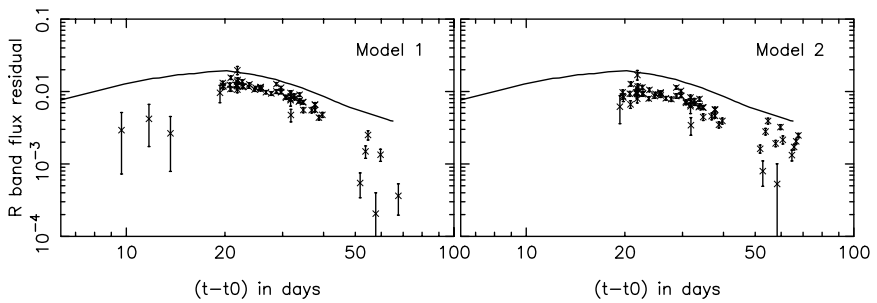

Fig. 5. $R$-band residuals for epochs beyond $\sim 7$ days, after subtracting the modeled flux of the afterglow and the contribution of the host galaxy $(R=22.6$, Gorosabel et al. 2005b) from the observed flux. The two models are shown in adjacent panels. This shows the $R$-band contribution needed from the associated supernova SN2003dh to explain the total observed light from the OT. The solid line is the red-shifted K-corrected SN1998bw $R$-band lightcurve, shown for comparison.

- We report several simultaneous two-band ( 90 and $\sim 250 \mathrm{GHz}$ ) detections of the afterglow for over a month after the burst.

- Our optical observations span a temporal range of $3 \mathrm{~h}$ to 34 days and fill in many gaps in the coverage reported so far in the literature. In $U B V I$ bands our data represent the earliest photometry of the optical transient.

- We find that the data can be well fit by a model where the two jets are present either simultaneously or in exclusion of each other. We derive a non-relativistic transition time of $\sim 42$ days for model 1 and $\sim 63$ days for model 2. Although concerns with reverse shock emission remain in both models, we deem it possible that the optical re-brightening seen at the epoch of $\sim 1.3$ days could be a re-energization of the jet, which resulted in the initially narrow jet being converted into a more energetic wider jet.

Acknowledgements. The GMRT is operated by the National Center For Radio Astrophysics of the Tata Institute of Fundamental Research. This work is based partly on observations carried out with the IRAM Plateau de Bure Interferometer. IRAM is supported by INSU/CNRS (France), MPG (Germany) and IGN (Spain). The SEST observations were conducted as part of the GRACE Collaboration Program for the multiwavelength follow up of Gamma-ray Bursts at ESO with ID 70.D-0523. We thank the ESO and IRAM Plateau de Bure staff and their operators for their great support in the observations. The HCT is operated by the Indian Institute of Astrophysics, Bangalore. This paper makes use of data obtained through the High Energy Astrophysics Science Archive Research Center Online Service, provided by the NASA/Goddard Space Flight Center. The GCN system, managed and operated by Scott Barthelmy, is gratefully acknowledged. This research is partially supported by the Spain's Ministerio de Educación y Ciencia through programs ESP2002-04124-C03-01 and AYA200401515 (including FEDER funds). LR acknowledges financial support from Council for Scientific and Industrial Research, India. The authors thank an anonymous referee for critical comments which helped improve the paper.

\section{References}

Band, D., Matteson, J., Ford, L., et al. 1993, ApJ, 413, 281

Berger, E., Kulkarni, S. R., Pooley, G., et al. 2003, Nature, 426, 154

Berger, E., Soderberg, A. M., \& Frail, D. A. 2003, GCNC, 2014

Booth, R., Delgado, G., Hagstrom, M., et al. 1989, A\&A, 216, 315
Frail, D. A., Waxman, E., \& Kulkarni, S. R. 2000, ApJ, 191

Galama, T. J., Vreeswijk, P. M., van Paradijs, J., et al. 1998, Nature, 395,670

Garnavich, P., Stanek, K. Z., \& Berlind, P. 2003, GCNC, 2018

Granot, J., Nakar, E., \& Piran, T. 2003, Nature, 426, 138 [arXiv: astro-ph/0304563]

Greiner, J., Peimbert, M., Estaban, C., et al. 2003a, GCNC, 2020

Greiner, J., Klose, S., Reinsch, K., et al. 2003b, Nature, 426, 157

Gorosabel, J., Castro-Tirado, A. J., Guziy, S., et al. 2005a, in preparation

Gorosabel, J., Ramírez-Pérez, M. D., Sollerman, J., et al. 2005b, A\&A, submitted

Guilloteau, S., Delannoy, J., Downes, et al. 1992, A\&A, 262, 624

Guziy, S., Castro-Tirado, A. J., Jóhannesson, G., et al. 2005, in preparation

Henden, A. 2003, GCNC, 2082

Hjorth, J., Sollerman, J., Møller, P., et al. 2003, Nature, 423, 847

Hoge, J. C., Meijerink, R., Tilanus, R. P. J., \& Smith, I. A. 2003, GCNC, 2088

Kobayashi, S. 2000, ApJ, 545, 807

Kumar, P., \& Piran, T. 2000, ApJ, 532, 286

Kuno, N., Sato, N., \& Nakanishi, H. 2003, GCNC, 2089

Lipkin, Y. M., Ofek, E. O., Gal-Yam, A., et al. 2004, ApJ, 606, L381

Marshall, F. E., \& Swank, J. H. 2003, GCNC, 1996

Matheson, T., Garnavich, P. M., Foltz, C., et al. 2003, ApJ, 582, L5

Oren, Y., Nakar, E., \& Piran, T. 2004 [arXiv: astro-ph/0406277]

Peng, F., Königl, A., \& Granot, J. 2004, ApJ, submitted [arXiv: astro-ph/0410384]

Peterson, B. A., \& Price, P. A. 2003, GCNC, 1985

Piran, T. 2005, Rev. Mod. Phys., 76, 1143

Piran, T., Nakar, E., \& Granot, J. 2003 [arXiv: astro-ph/0312138], Talk given by T. Piran at the GRB Symposium, Santa Fe, September, 2003

Pooley, G. 2003, GCNC, 2043

Price, P. A., Fox, D. W., Kulkarni, S. R., et al. 2003, Nature, 423, 844

Rao, A. P., Ishwara-Chandra, C. H., \& Bhattacharya, D. 2003a, GCNC, 2073

Rao, A. P., Ishwara-Chandra, C. H., Bhattacharya, D., \& Castro-Tirado, A. J. 2003b, GCNC, 2268

Rhoads, J. E. 1999, ApJ, 525, 737

Sagar, R. 2002, BASI, 30, 237

Sari, R., \& Mészáros, P. 2000, ApJ, 535, L33

Sari, R., \& Piran, T. 1995, ApJ, 455, L143

Sari, R., Piran, T., \& Halpern, J. P. 1999, ApJ, 590, L17

Sato, R., Kawai, N., Suzuki, M., et al. 2003, ApJ, 599, L9

Schlegel, D. J., Finkbeiner, D. P., \& Davis, M. 1998, ApJ, 500, 525

Sheth, K., Frail, D. A., White, S., et al. 2003, ApJ, 595, L33

Smith, D. A. 2003, GCNC, 2019

Stanek, K. Z., Matheson, T., Garnavich, P. M., \& Martini, P. 2003, ApJ, 591, L17

Swarup, G., Ananthakrishnan, S., Kapahi, V. K., et al. 1991, Curr. Sci., 60,95

Taylor, G. B., Frail, D. A., Berger, E., \& Kulkarni, S. R. 2004, ApJ, 609, L1

Tiengo, A., Mereghetti, S., Ghisellini, G., et al. 2003, A\&A, 409, 983

Tiengo, A., Mereghetti, S., Ghisellini, G., Tavecchio, F., \& Ghirlanda, G. 2004, A\&A, 423, 861

Torii, K. 2003, GCNC, 1986

Vanderspek, R., Crew, G., Doty, J., et al. 2003a, GCNC, 1997

Vanderspek, R., Sakamoto, T., Barraud, C., et al. 2004, ApJ, 617, 1251

Wijers, R. A. M. J., \& Galama, T. J. 1999, ApJ, 523, 177 


\section{Online Material}


L. Resmi et al.: Radio, millimeter and optical afterglow of GRB 030329, Online Material p 2

Table 2. Optical transient.

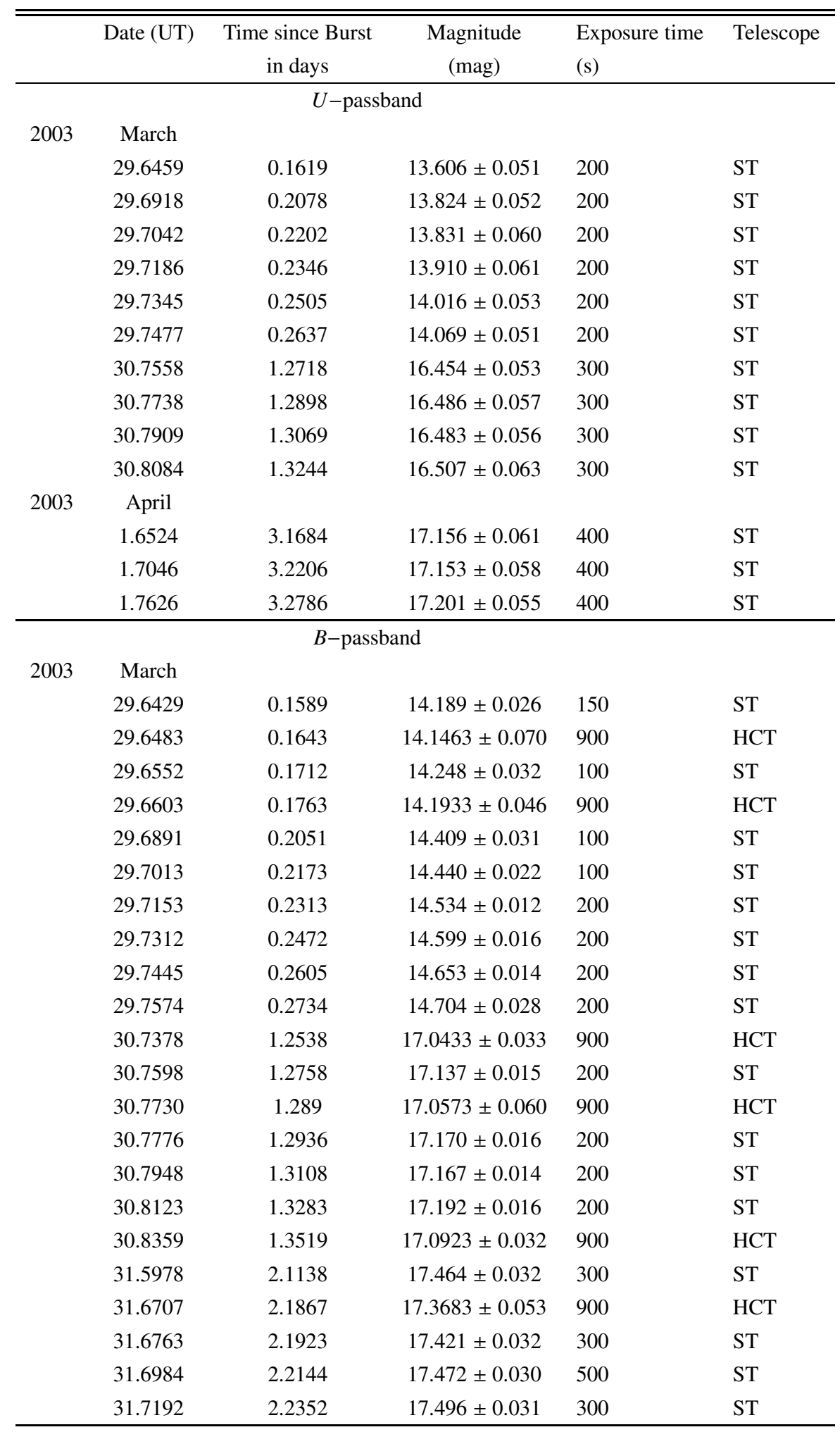


L. Resmi et al.: Radio, millimeter and optical afterglow of GRB 030329, Online Material p 3

Table 2. continued.

\begin{tabular}{|c|c|c|c|c|c|}
\hline & Date (UT) & $\begin{array}{c}\text { Time since Burst } \\
\text { in days }\end{array}$ & $\begin{array}{l}\text { Magnitude } \\
\text { (mag) }\end{array}$ & $\begin{array}{l}\text { Exposure time } \\
\text { (s) }\end{array}$ & Telescope \\
\hline \multirow{41}{*}{2003} & 31.7410 & 2.257 & $17.4503 \pm 0.028$ & 900 & HCT \\
\hline & 31.7414 & 2.2574 & $17.509 \pm 0.030$ & 300 & ST \\
\hline & 31.7598 & 2.2758 & $17.508 \pm 0.029$ & 300 & ST \\
\hline & 31.7740 & 2.29 & $17.4403 \pm 0.029$ & 900 & HCT \\
\hline & 31.7769 & 2.2929 & $17.485 \pm 0.031$ & 300 & ST \\
\hline & 31.7970 & 2.313 & $17.520 \pm 0.029$ & 300 & ST \\
\hline & 31.8046 & 2.3206 & $17.4553 \pm 0.020$ & 900 & HCT \\
\hline & 31.8149 & 2.3309 & $17.550 \pm 0.028$ & 400 & ST \\
\hline & 31.8343 & 2.3503 & $17.4853 \pm 0.028$ & 900 & HCT \\
\hline & 31.8387 & 2.3547 & $17.586 \pm 0.029$ & 400 & ST \\
\hline & 31.8600 & 2.376 & $17.595 \pm 0.031$ & 400 & ST \\
\hline & 31.8806 & 2.3966 & $17.617 \pm 0.022$ & 400 & ST \\
\hline & April & & & & \\
\hline & 1.5967 & 3.1127 & $17.866 \pm 0.033$ & 300 & ST \\
\hline & 1.6277 & 3.1437 & $17.749 \pm 0.038$ & 200 & ST \\
\hline & 1.7153 & 3.2313 & $17.826 \pm 0.029$ & 400 & ST \\
\hline & 1.7207 & 3.2367 & $17.831 \pm 0.027$ & 400 & ST \\
\hline & 1.7356 & 3.2516 & $17.7193 \pm 0.031$ & 900 & HCT \\
\hline & 1.8582 & 3.3742 & $17.791 \pm 0.037$ & 300 & ST \\
\hline & 1.9000 & 3.416 & $17.7843 \pm 0.034$ & 900 & HCT \\
\hline & 2.6386 & 4.1546 & $18.3483 \pm 0.036$ & 900 & HCT \\
\hline & 2.6436 & 4.1596 & $18.419 \pm 0.041$ & 300 & ST \\
\hline & 2.6771 & 4.1931 & $18.4003 \pm 0.029$ & 900 & HCT \\
\hline & 2.6862 & 4.2022 & $18.534 \pm 0.039$ & 300 & ST \\
\hline & 2.7439 & 4.2599 & $18.528 \pm 0.038$ & 300 & ST \\
\hline & 2.7707 & 4.2867 & $18.506 \pm 0.039$ & 300 & ST \\
\hline & 4.6673 & 6.1833 & $18.787 \pm 0.039$ & 400 & ST \\
\hline & 4.6966 & 6.2126 & $18.748 \pm 0.034$ & 400 & ST \\
\hline & 4.7246 & 6.2406 & $18.852 \pm 0.033$ & 400 & ST \\
\hline & 5.6125 & 7.1285 & $19.2573 \pm 0.067$ & 900 & HCT \\
\hline & 5.6511 & 7.1671 & $19.2373 \pm 0.053$ & 900 & HCT \\
\hline & 5.7416 & 7.2576 & $19.2963 \pm 0.046$ & 900 & HCT \\
\hline & 5.7702 & 7.2862 & $19.3183 \pm 0.044$ & 900 & HCT \\
\hline & 5.8037 & 7.3197 & $19.3153 \pm 0.046$ & 900 & HCT \\
\hline & 5.8293 & 7.3453 & $19.3683 \pm 0.030$ & 900 & HCT \\
\hline & 5.8618 & 7.3778 & $19.3873 \pm 0.045$ & 900 & HCT \\
\hline & 6.6438 & 8.1598 & $19.6213 \pm 0.027$ & 900 & HCT \\
\hline & 6.6559 & 8.1719 & $19.6443 \pm 0.028$ & 900 & $\mathrm{HCT}$ \\
\hline & 6.7522 & 8.2682 & $19.6063 \pm 0.030$ & 900 & HCT \\
\hline & 6.8384 & 8.3544 & $19.6183 \pm 0.039$ & 900 & HCT \\
\hline & 6.8505 & 8.3665 & $19.6553 \pm 0.034$ & 900 & HCT \\
\hline
\end{tabular}


Table 2. continued.

\begin{tabular}{|c|c|c|c|c|c|}
\hline & Date (UT) & $\begin{array}{c}\text { Time since Burst } \\
\text { in days }\end{array}$ & $\begin{array}{l}\text { Magnitude } \\
\text { (mag) }\end{array}$ & $\begin{array}{l}\text { Exposure time } \\
\text { (s) }\end{array}$ & Telescope \\
\hline \multirow{9}{*}{2003} & 7.6122 & 9.1282 & $19.761 \pm 0.144$ & 400 & $\mathrm{ST}$ \\
\hline & 8.6260 & 10.142 & $19.983 \pm 0.069$ & 600 & $\mathrm{ST}$ \\
\hline & 8.6641 & 10.1801 & $19.943 \pm 0.076$ & 600 & $\mathrm{ST}$ \\
\hline & 8.6933 & 10.2093 & $19.919 \pm 0.064$ & 600 & ST \\
\hline & 10.6368 & 12.1528 & $20.287 \pm 0.087$ & 900 & $\mathrm{ST}$ \\
\hline & 10.7059 & 12.2219 & $20.1933 \pm 0.030$ & $600 \times 4$ & HCT \\
\hline & 11.6782 & 13.1942 & $20.432 \pm 0.177$ & 600 & ST \\
\hline & May & & & & \\
\hline & 1.6271 & 33.1431 & $22.214 \pm 0.156$ & $3 * 500+900$ & $\mathrm{ST}$ \\
\hline \multicolumn{6}{|c|}{$V$-passband } \\
\hline \multirow[t]{30}{*}{2003} & March & & & & \\
\hline & 29.6310 & 0.147 & $13.8983 \pm 0.029$ & 600 & HCT \\
\hline & 29.6363 & 0.1523 & $13.848 \pm 0.020$ & 200 & ST \\
\hline & 29.6515 & 0.1675 & $13.930 \pm 0.023$ & 75 & ST \\
\hline & 29.6872 & 0.2032 & $14.087 \pm 0.021$ & 50 & $\mathrm{ST}$ \\
\hline & 29.6991 & 0.2151 & $14.142 \pm 0.017$ & 100 & ST \\
\hline & 29.7127 & 0.2287 & $14.213 \pm 0.018$ & 100 & ST \\
\hline & 29.7284 & 0.2444 & $14.266 \pm 0.033$ & 100 & ST \\
\hline & 29.7416 & 0.2576 & $14.331 \pm 0.018$ & 100 & ST \\
\hline & 29.7552 & 0.2712 & $14.371 \pm 0.032$ & 100 & ST \\
\hline & 29.7985 & 0.3145 & $14.569 \pm 0.032$ & 200 & $\mathrm{ST}$ \\
\hline & 29.8773 & 0.3933 & $14.9323 \pm 0.050$ & 600 & HCT \\
\hline & 30.6674 & 1.1834 & $16.7423 \pm 0.047$ & 600 & HCT \\
\hline & 30.7486 & 1.2646 & $16.7943 \pm 0.050$ & 600 & HCT \\
\hline & 30.7632 & 1.2792 & $16.790 \pm 0.013$ & 200 & ST \\
\hline & 30.7809 & 1.2969 & $16.789 \pm 0.013$ & 200 & ST \\
\hline & 30.7836 & 1.2996 & $16.8153 \pm 0.032$ & 600 & HCT \\
\hline & 30.7980 & 1.314 & $16.773 \pm 0.013$ & 200 & ST \\
\hline & 30.8202 & 1.3362 & $16.825 \pm 0.013$ & 200 & ST \\
\hline & 30.8256 & 1.3416 & $16.8363 \pm 0.040$ & 600 & HCT \\
\hline & 30.8680 & 1.384 & $16.8183 \pm 0.046$ & 600 & HCT \\
\hline & 30.8998 & 1.4158 & $16.8353 \pm 0.050$ & 600 & HCT \\
\hline & 31.6026 & 2.1186 & $17.111 \pm 0.013$ & 300 & $\mathrm{ST}$ \\
\hline & 31.6531 & 2.1691 & $17.1033 \pm 0.064$ & 600 & $\mathrm{HCT}$ \\
\hline & 31.6811 & 2.1971 & $17.094 \pm 0.024$ & 300 & ST \\
\hline & 31.7041 & 2.2201 & $17.126 \pm 0.018$ & 300 & ST \\
\hline & 31.7263 & 2.2423 & $17.148 \pm 0.017$ & 300 & $\mathrm{ST}$ \\
\hline & 31.7308 & 2.2468 & $17.1783 \pm 0.055$ & 600 & HCT \\
\hline & 31.7459 & 2.2619 & $17.150 \pm 0.016$ & 300 & $\mathrm{ST}$ \\
\hline & 31.7589 & 2.2749 & $17.1653 \pm 0.052$ & 600 & HCT \\
\hline
\end{tabular}


L. Resmi et al.: Radio, millimeter and optical afterglow of GRB 030329, Online Material p 5

Table 2. continued.

\begin{tabular}{|c|c|c|c|c|c|}
\hline & Date (UT) & $\begin{array}{c}\text { Time since Burst } \\
\text { in days }\end{array}$ & $\begin{array}{l}\text { Magnitude } \\
\text { (mag) }\end{array}$ & $\begin{array}{l}\text { Exposure time } \\
\text { (s) }\end{array}$ & Telescope \\
\hline \multirow{40}{*}{2003} & 31.7643 & 2.2803 & $17.148 \pm 0.018$ & 300 & ST \\
\hline & 31.7814 & 2.2974 & $17.157 \pm 0.020$ & 300 & $\mathrm{ST}$ \\
\hline & 31.7894 & 2.3054 & $17.1883 \pm 0.040$ & 600 & HCT \\
\hline & 31.8015 & 2.3175 & $17.179 \pm 0.014$ & 300 & ST \\
\hline & 31.8211 & 2.3371 & $17.212 \pm 0.014$ & 400 & $\mathrm{ST}$ \\
\hline & 31.8216 & 2.3376 & $17.2143 \pm 0.062$ & 600 & HCT \\
\hline & 31.8444 & 2.3604 & $17.227 \pm 0.016$ & 400 & ST \\
\hline & 31.8498 & 2.3658 & $17.2443 \pm 0.069$ & 600 & HCT \\
\hline & 31.8656 & 2.3816 & $17.253 \pm 0.014$ & 400 & $\mathrm{ST}$ \\
\hline & 31.8781 & 2.3941 & $17.2633 \pm 0.055$ & 600 & HCT \\
\hline & 31.8862 & 2.4022 & $17.253 \pm 0.020$ & 400 & ST \\
\hline & 31.9177 & 2.4337 & $17.2843 \pm 0.059$ & 600 & HCT \\
\hline & April & & & & \\
\hline & 1.6139 & 3.1299 & $17.438 \pm 0.017$ & 300 & $\mathrm{ST}$ \\
\hline & 1.6727 & 3.1887 & $17.453 \pm 0.017$ & 300 & $\mathrm{ST}$ \\
\hline & 1.6758 & 3.1918 & $17.4463 \pm 0.051$ & 450 & HCT \\
\hline & 1.7215 & 3.2375 & $17.4203 \pm 0.051$ & 450 & HCT \\
\hline & 1.7326 & 3.2486 & $17.453 \pm 0.016$ & 400 & ST \\
\hline & 1.7381 & 3.2541 & $17.464 \pm 0.016$ & 400 & ST \\
\hline & 1.8841 & 3.4001 & $17.427 \pm 0.018$ & 300 & ST \\
\hline & 1.8847 & 3.4007 & $17.4233 \pm 0.040$ & 450 & HCT \\
\hline & 2.6234 & 4.1394 & $18.0493 \pm 0.061$ & 450 & HCT \\
\hline & 2.6595 & 4.1755 & $18.048 \pm 0.021$ & 300 & ST \\
\hline & 2.6612 & 4.1772 & $18.0873 \pm 0.065$ & 450 & HCT \\
\hline & 2.6903 & 4.2063 & $18.0913 \pm 0.064$ & 450 & HCT \\
\hline & 2.6909 & 4.2069 & $18.068 \pm 0.020$ & 300 & ST \\
\hline & 2.7221 & 4.2381 & $18.112 \pm 0.024$ & 300 & ST \\
\hline & 4.6767 & 6.1927 & $18.370 \pm 0.024$ & 400 & ST \\
\hline & 4.7069 & 6.2229 & $18.393 \pm 0.025$ & 400 & ST \\
\hline & 4.7302 & 6.2462 & $18.481 \pm 0.023$ & 400 & ST \\
\hline & 5.5977 & 7.1137 & $18.8733 \pm 0.036$ & 450 & HCT \\
\hline & 5.6670 & 7.183 & $18.8953 \pm 0.039$ & 450 & HCT \\
\hline & 5.6997 & 7.2157 & $18.9383 \pm 0.052$ & 450 & HCT \\
\hline & 5.7303 & 7.2463 & $18.9283 \pm 0.055$ & 450 & HCT \\
\hline & 5.7573 & 7.2733 & $18.9353 \pm 0.044$ & 450 & HCT \\
\hline & 6.6149 & 8.1309 & $19.1813 \pm 0.027$ & $600 \times 2$ & HCT \\
\hline & 6.7218 & 8.2378 & $19.2393 \pm 0.027$ & $600 \times 3$ & HCT \\
\hline & 6.8111 & 8.3271 & $19.2183 \pm 0.046$ & $600 \times 3$ & HCT \\
\hline & 6.8767 & 8.3927 & $19.2253 \pm 0.064$ & $600 \times 2$ & HCT \\
\hline & 7.6073 & 9.1233 & $19.294 \pm 0.073$ & 300 & ST \\
\hline
\end{tabular}


Table 2. continued.

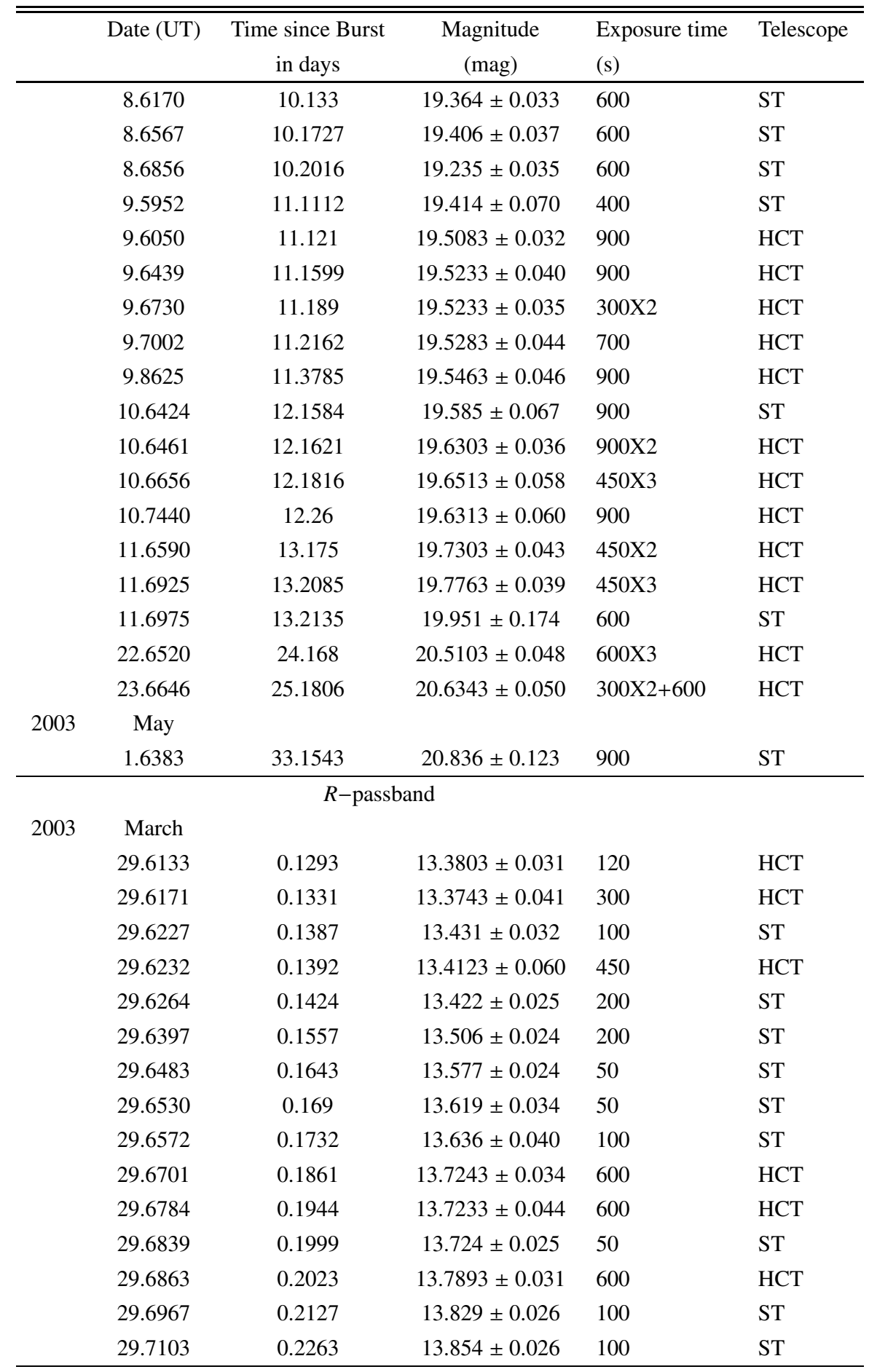


L. Resmi et al.: Radio, millimeter and optical afterglow of GRB 030329, Online Material p 7

Table 2. continued.

\begin{tabular}{|c|c|c|c|c|}
\hline Date (UT) & $\begin{array}{c}\text { Time since Burst } \\
\text { in days }\end{array}$ & $\begin{array}{l}\text { Magnitude } \\
\text { (mag) }\end{array}$ & $\begin{array}{l}\text { Exposure time } \\
\text { (s) }\end{array}$ & Telescope \\
\hline 29.7262 & 0.2422 & $13.916 \pm 0.026$ & 100 & ST \\
\hline 29.7396 & 0.2556 & $13.982 \pm 0.027$ & 100 & ST \\
\hline 29.7526 & 0.2686 & $14.030 \pm 0.027$ & 100 & ST \\
\hline 29.7650 & 0.281 & $14.098 \pm 0.052$ & 200 & ST \\
\hline 29.7937 & 0.3097 & $14.210 \pm 0.032$ & 100 & ST \\
\hline 29.8675 & 0.3835 & $14.4893 \pm 0.050$ & 600 & HCT \\
\hline 29.8857 & 0.4017 & $14.5483 \pm 0.046$ & 600 & HCT \\
\hline 29.9079 & 0.4239 & $14.6333 \pm 0.032$ & 450 & HCT \\
\hline 29.9140 & 0.43 & $14.6533 \pm 0.033$ & 450 & HCT \\
\hline 29.9201 & 0.4361 & $14.6613 \pm 0.040$ & 450 & HCT \\
\hline 29.9275 & 0.4435 & $14.6753 \pm 0.070$ & 450 & HCT \\
\hline 30.5833 & 1.0993 & $16.249 \pm 0.070$ & 100 & ST \\
\hline 30.5875 & 1.1035 & $16.295 \pm 0.056$ & 200 & ST \\
\hline 30.6518 & 1.1678 & $16.2963 \pm 0.070$ & 180 & HCT \\
\hline 30.6586 & 1.1746 & $16.3343 \pm 0.042$ & 600 & HCT \\
\hline 30.6747 & 1.1907 & $16.3533 \pm 0.039$ & 450 & HCT \\
\hline 30.6879 & 1.2039 & $16.3733 \pm 0.028$ & 450 & HCT \\
\hline 30.7268 & 1.2428 & $16.3723 \pm 0.049$ & 450 & HCT \\
\hline 30.7637 & 1.2797 & $16.4133 \pm 0.040$ & 450 & HCT \\
\hline 30.7665 & 1.2825 & $16.420 \pm 0.021$ & 200 & ST \\
\hline 30.7841 & 1.3001 & $16.438 \pm 0.018$ & 200 & ST \\
\hline 30.7971 & 1.3131 & $16.4223 \pm 0.050$ & 450 & HCT \\
\hline 30.8012 & 1.3172 & $16.439 \pm 0.018$ & 200 & ST \\
\hline 30.8176 & 1.3336 & $16.4453 \pm 0.041$ & 450 & HCT \\
\hline 30.8238 & 1.3398 & $16.460 \pm 0.017$ & 200 & ST \\
\hline 30.8457 & 1.3617 & $16.4593 \pm 0.039$ & 450 & HCT \\
\hline 30.8603 & 1.3763 & $16.4683 \pm 0.034$ & 450 & HCT \\
\hline 30.8759 & 1.3919 & $16.4623 \pm 0.032$ & 450 & HCT \\
\hline 30.8914 & 1.4074 & $16.4613 \pm 0.039$ & 450 & HCT \\
\hline 30.9076 & 1.4236 & $16.4353 \pm 0.057$ & 450 & HCT \\
\hline 30.9142 & 1.4302 & $16.4703 \pm 0.034$ & 450 & HCT \\
\hline 30.9212 & 1.4372 & $16.4303 \pm 0.031$ & 450 & HCT \\
\hline 30.9276 & 1.4436 & $16.4013 \pm 0.063$ & 450 & HCT \\
\hline 31.6449 & 2.1609 & $16.7163 \pm 0.040$ & 450 & HCT \\
\hline 31.6610 & 2.177 & $16.7163 \pm 0.031$ & 450 & HCT \\
\hline 31.6653 & 2.1813 & $16.711 \pm 0.032$ & 100 & ST \\
\hline 31.6804 & 2.1964 & $16.7103 \pm 0.041$ & 450 & HCT \\
\hline 31.6898 & 2.2058 & $16.744 \pm 0.033$ & 200 & ST \\
\hline
\end{tabular}


Table 2. continued.

\begin{tabular}{|c|c|c|c|c|c|}
\hline & Date (UT) & $\begin{array}{c}\text { Time since Burst } \\
\text { in days }\end{array}$ & $\begin{array}{l}\text { Magnitude } \\
\text { (mag) }\end{array}$ & $\begin{array}{l}\text { Exposure time } \\
\text { (s) }\end{array}$ & Telescope \\
\hline & 31.7083 & 2.2243 & $16.771 \pm 0.031$ & 200 & $\mathrm{ST}$ \\
\hline & 31.7148 & 2.2308 & $16.783 \pm 0.027$ & 300 & $\mathrm{ST}$ \\
\hline & 31.7231 & 2.2391 & $16.7823 \pm 0.026$ & 450 & HCT \\
\hline & 31.7303 & 2.2463 & $16.772 \pm 0.027$ & 200 & $\mathrm{ST}$ \\
\hline & 31.7500 & 2.266 & $16.779 \pm 0.026$ & 200 & ST \\
\hline & 31.7507 & 2.2667 & $16.7953 \pm 0.040$ & 450 & HCT \\
\hline & 31.7655 & 2.2815 & $16.7933 \pm 0.035$ & 300 & HCT \\
\hline & 31.7682 & 2.2842 & $16.788 \pm 0.029$ & 200 & ST \\
\hline & 31.7826 & 2.2986 & $16.7953 \pm 0.043$ & 300 & HCT \\
\hline & 31.7853 & 2.3013 & $16.798 \pm 0.029$ & 200 & ST \\
\hline & 31.7943 & 2.3103 & $16.8203 \pm 0.025$ & 300 & HCT \\
\hline & 31.8054 & 2.3214 & $16.820 \pm 0.025$ & 200 & ST \\
\hline & 31.8139 & 2.3299 & $16.8083 \pm 0.034$ & 300 & HCT \\
\hline & 31.8264 & 2.3424 & $16.8443 \pm 0.043$ & 300 & HCT \\
\hline & 31.8280 & 2.344 & $16.844 \pm 0.024$ & 300 & ST \\
\hline & 31.8431 & 2.3591 & $16.8503 \pm 0.040$ & 300 & HCT \\
\hline & 31.8495 & 2.3655 & $16.867 \pm 0.024$ & 300 & $\mathrm{ST}$ \\
\hline & 31.8545 & 2.3705 & $16.8583 \pm 0.048$ & 300 & HCT \\
\hline & 31.8705 & 2.3865 & $16.874 \pm 0.024$ & 300 & $\mathrm{ST}$ \\
\hline & 31.8713 & 2.3873 & $16.8573 \pm 0.044$ & 300 & HCT \\
\hline & 31.8832 & 2.3992 & $16.8683 \pm 0.045$ & 300 & HCT \\
\hline & 31.8912 & 2.4072 & $16.900 \pm 0.023$ & 300 & ST \\
\hline & 31.8999 & 2.4159 & $16.8723 \pm 0.059$ & 300 & HCT \\
\hline & 31.9111 & 2.4271 & $16.9013 \pm 0.037$ & 300 & HCT \\
\hline & 31.9223 & 2.4383 & $16.9163 \pm 0.028$ & 300 & HCT \\
\hline \multirow[t]{15}{*}{2003} & April & & & & \\
\hline & 1.6008 & 3.1168 & $17.071 \pm 0.027$ & 200 & ST \\
\hline & 1.6237 & 3.1397 & $17.045 \pm 0.025$ & 300 & ST \\
\hline & 1.6569 & 3.1729 & $17.059 \pm 0.025$ & 200 & $\mathrm{ST}$ \\
\hline & 1.6694 & 3.1854 & $17.0553 \pm 0.046$ & 300 & HCT \\
\hline & 1.6766 & 3.1926 & $17.075 \pm 0.026$ & 200 & ST \\
\hline & 1.6821 & 3.1981 & $17.0453 \pm 0.045$ & 300 & HCT \\
\hline & 1.6950 & 3.211 & $17.079 \pm 0.026$ & 200 & ST \\
\hline & 1.7274 & 3.2434 & $17.0473 \pm 0.037$ & 300 & HCT \\
\hline & 1.7445 & 3.2605 & $17.0583 \pm 0.032$ & 300 & HCT \\
\hline & 1.7487 & 3.2647 & $17.075 \pm 0.029$ & 300 & ST \\
\hline & 1.7531 & 3.2691 & $17.071 \pm 0.026$ & 300 & ST \\
\hline & 1.8628 & 3.3788 & $17.058 \pm 0.023$ & 300 & ST \\
\hline & 1.8891 & 3.4051 & $17.039 \pm 0.024$ & 300 & $\mathrm{ST}$ \\
\hline & 1.8906 & 3.4066 & $17.0273 \pm 0.035$ & 300 & HCT \\
\hline
\end{tabular}


L. Resmi et al.: Radio, millimeter and optical afterglow of GRB 030329, Online Material p 9

Table 2. continued.

\begin{tabular}{|c|c|c|c|c|}
\hline Date (UT) & $\begin{array}{c}\text { Time since Burst } \\
\text { in days }\end{array}$ & $\begin{array}{c}\text { Magnitude } \\
\text { (mag) }\end{array}$ & $\begin{array}{l}\text { Exposure time } \\
\text { (s) }\end{array}$ & Telescope \\
\hline 1.9103 & 3.4263 & $17.101 \pm 0.028$ & 300 & ST \\
\hline 2.6158 & 4.1318 & $17.6723 \pm 0.046$ & 300 & HCT \\
\hline 2.6391 & 4.1551 & $17.694 \pm 0.028$ & 300 & ST \\
\hline 2.6425 & 4.1585 & $17.6933 \pm 0.029$ & 300 & HCT \\
\hline 2.6531 & 4.1691 & $17.7113 \pm 0.029$ & 300 & HCT \\
\hline 2.6553 & 4.1713 & $17.707 \pm 0.025$ & 300 & ST \\
\hline 2.6697 & 4.1857 & $17.680 \pm 0.027$ & 300 & ST \\
\hline 2.6843 & 4.2003 & $17.7193 \pm 0.033$ & 300 & HCT \\
\hline 2.6953 & 4.2113 & $17.689 \pm 0.028$ & 300 & ST \\
\hline 2.6962 & 4.2122 & $17.7023 \pm 0.033$ & 300 & HCT \\
\hline 2.7124 & 4.2284 & $17.706 \pm 0.034$ & 300 & ST \\
\hline 2.7265 & 4.2425 & $17.709 \pm 0.030$ & 300 & ST \\
\hline 2.7484 & 4.2644 & $17.721 \pm 0.030$ & 300 & ST \\
\hline 2.7615 & 4.2775 & $17.737 \pm 0.030$ & 300 & ST \\
\hline 2.7752 & 4.2912 & $17.718 \pm 0.032$ & 300 & ST \\
\hline 4.6831 & 6.1991 & $18.030 \pm 0.031$ & 400 & ST \\
\hline 4.7125 & 6.2285 & $18.085 \pm 0.035$ & 400 & ST \\
\hline 4.7357 & 6.2517 & $18.130 \pm 0.031$ & 400 & ST \\
\hline 5.5919 & 7.1079 & $18.4583 \pm 0.049$ & 300 & HCT \\
\hline 5.6044 & 7.1204 & $18.5093 \pm 0.028$ & 300 & $\mathrm{HCT}$ \\
\hline 5.6209 & 7.1369 & $18.5033 \pm 0.025$ & 300 & HCT \\
\hline 5.6340 & 7.15 & $18.4963 \pm 0.032$ & 300 & $\mathrm{HCT}$ \\
\hline 5.6603 & 7.1763 & $18.4803 \pm 0.036$ & 300 & HCT \\
\hline 5.6858 & 7.2018 & $18.5763 \pm 0.029$ & 300 & HCT \\
\hline 5.7054 & 7.2214 & $18.5873 \pm 0.024$ & 300 & HCT \\
\hline 5.7231 & 7.2391 & $18.5243 \pm 0.029$ & 300 & HCT \\
\hline 5.7507 & 7.2667 & $18.5683 \pm 0.027$ & 300 & HCT \\
\hline 5.7790 & 7.295 & $18.5873 \pm 0.027$ & 300 & HCT \\
\hline 5.7949 & 7.3109 & $18.5803 \pm 0.036$ & 300 & HCT \\
\hline 5.8130 & 7.329 & $18.6003 \pm 0.027$ & 300 & HCT \\
\hline 5.8454 & 7.3614 & $18.5893 \pm 0.035$ & 300 & HCT \\
\hline 5.8704 & 7.3864 & $18.6283 \pm 0.037$ & 300 & HCT \\
\hline 5.9002 & 7.4162 & $18.6643 \pm 0.049$ & 300 & HCT \\
\hline 6.5919 & 8.1079 & $18.8233 \pm 0.039$ & 300 & HCT \\
\hline 6.5995 & 8.1155 & $18.8373 \pm 0.029$ & 300 & HCT \\
\hline 6.6234 & 8.1394 & $18.8053 \pm 0.036$ & 300 & HCT \\
\hline 6.6849 & 8.2009 & $18.8453 \pm 0.025$ & 600 & HCT \\
\hline 6.6932 & 8.2092 & $18.8503 \pm 0.030$ & 600 & HCT \\
\hline
\end{tabular}


L. Resmi et al.: Radio, millimeter and optical afterglow of GRB 030329, Online Material p 10

Table 2. continued.

\begin{tabular}{|c|c|c|c|c|c|}
\hline & Date (UT) & $\begin{array}{c}\text { Time since Burst } \\
\text { in days }\end{array}$ & $\begin{array}{l}\text { Magnitude } \\
\text { (mag) }\end{array}$ & $\begin{array}{l}\text { Exposure time } \\
\text { (s) }\end{array}$ & Telescope \\
\hline & 6.7327 & 8.2487 & $18.8503 \pm 0.032$ & 600 & HCT \\
\hline & 6.7413 & 8.2573 & $18.8443 \pm 0.022$ & 600 & HCT \\
\hline & 6.7792 & 8.2952 & $18.8363 \pm 0.029$ & 600 & HCT \\
\hline & 6.7878 & 8.3038 & $18.8433 \pm 0.034$ & 600 & HCT \\
\hline & 6.8205 & 8.3365 & $18.8523 \pm 0.029$ & 600 & HCT \\
\hline & 6.8286 & 8.3446 & $18.8473 \pm 0.047$ & 600 & HCT \\
\hline & 6.8851 & 8.4011 & $18.8823 \pm 0.043$ & 600 & HCT \\
\hline & 6.8935 & 8.4095 & $18.8773 \pm 0.038$ & 600 & HCT \\
\hline & 6.9016 & 8.4176 & $18.8183 \pm 0.031$ & 600 & HCT \\
\hline & 7.5936 & 9.1096 & $18.897 \pm 0.052$ & 300 & ST \\
\hline & 7.5980 & 9.114 & $18.945 \pm 0.067$ & 300 & ST \\
\hline & 8.6070 & 10.123 & $19.017 \pm 0.046$ & 400 & ST \\
\hline & 8.6483 & 10.1643 & $19.049 \pm 0.041$ & 600 & ST \\
\hline & 8.6778 & 10.1938 & $19.064 \pm 0.032$ & 600 & ST \\
\hline & 9.5890 & 11.105 & $19.078 \pm 0.057$ & 400 & ST \\
\hline & 9.5905 & 11.1065 & $19.1263 \pm 0.033$ & 900 & HCT \\
\hline & 9.6321 & 11.1481 & $19.0963 \pm 0.037$ & 900 & $\mathrm{HCT}$ \\
\hline & 9.6875 & 11.2035 & $19.1403 \pm 0.037$ & $300 \times 2$ & $\mathrm{HCT}$ \\
\hline & 9.7242 & 11.2402 & $19.1743 \pm 0.039$ & $300 \times 3$ & $\mathrm{HCT}$ \\
\hline & 9.8502 & 11.3662 & $19.1663 \pm 0.050$ & 900 & HCT \\
\hline & 10.6068 & 12.1228 & $19.3113 \pm 0.030$ & 900 & HCT \\
\hline & 10.6157 & 12.1317 & $19.440 \pm 0.095$ & 400 & ST \\
\hline & 10.6437 & 12.1597 & $19.2923 \pm 0.052$ & 900 & HCT \\
\hline & 10.6483 & 12.1643 & $19.294 \pm 0.057$ & 900 & ST \\
\hline & 10.6825 & 12.1985 & $19.2553 \pm 0.050$ & $300 \times 3$ & HCT \\
\hline & 10.7272 & 12.2432 & $19.3483 \pm 0.039$ & $300+450$ & HCT \\
\hline & 10.7390 & 12.255 & $19.3503 \pm 0.041$ & 300 & HCT \\
\hline & 10.7574 & 12.2734 & $19.3283 \pm 0.049$ & 900 & $\mathrm{HCT}$ \\
\hline & 10.8689 & 12.3849 & $19.3463 \pm 0.060$ & 900 & HCT \\
\hline & 11.6743 & 13.1903 & $19.4273 \pm 0.038$ & $300 \times 3$ & HCT \\
\hline & 11.7064 & 13.2224 & $19.527 \pm 0.154$ & 600 & ST \\
\hline & 11.7082 & 13.2242 & $19.2813 \pm 0.053$ & $300 \times 2$ & HCT \\
\hline & 22.6028 & 24.1188 & $20.1233 \pm 0.043$ & 600 & HCT \\
\hline & 22.6279 & 24.1439 & $20.0573 \pm 0.040$ & $600 \times 2$ & HCT \\
\hline & 22.8033 & 24.3193 & $20.0633 \pm 0.025$ & $600 \times 3$ & $\mathrm{HCT}$ \\
\hline \multirow{3}{*}{2003} & 23.6311 & 25.1471 & $20.0983 \pm 0.032$ & $300 \times 5$ & $\mathrm{HCT}$ \\
\hline & May & & & & \\
\hline & 1.6069 & 33.1229 & $\pm 20.692 \pm 0.090$ & $2 * 300$ & ST \\
\hline
\end{tabular}


Table 2. continued.

\begin{tabular}{|c|c|c|c|c|c|}
\hline & Date (UT) & $\begin{array}{c}\text { Time since Burst } \\
\text { in days }\end{array}$ & $\begin{array}{l}\text { Magnitude } \\
\text { (mag) }\end{array}$ & $\begin{array}{l}\text { Exposure time } \\
\text { (s) }\end{array}$ & Telescope \\
\hline \multicolumn{6}{|c|}{$I$-passband } \\
\hline \multirow[t]{27}{*}{2003} & March & & & & \\
\hline & 29.6306 & 0.1466 & $13.037 \pm 0.026$ & 200 & ST \\
\hline & 29.6499 & 0.1659 & $13.185 \pm 0.029$ & 50 & ST \\
\hline & 29.6855 & 0.2015 & $13.328 \pm 0.029$ & 50 & ST \\
\hline & 29.6948 & 0.2108 & $13.487 \pm 0.031$ & 50 & ST \\
\hline & 29.7083 & 0.2243 & $13.437 \pm 0.030$ & 100 & ST \\
\hline & 29.7241 & 0.2401 & $13.501 \pm 0.032$ & 100 & ST \\
\hline & 29.7374 & 0.2534 & $13.559 \pm 0.032$ & 100 & ST \\
\hline & 29.7504 & 0.2664 & $13.618 \pm 0.032$ & 100 & ST \\
\hline & 29.7616 & 0.2776 & $13.685 \pm 0.032$ & 200 & ST \\
\hline & 29.7959 & 0.3119 & $13.837 \pm 0.049$ & 100 & ST \\
\hline & 30.7698 & 1.2858 & $15.972 \pm 0.023$ & 200 & ST \\
\hline & 30.7872 & 1.3032 & $15.985 \pm 0.021$ & 200 & ST \\
\hline & 30.8046 & 1.3206 & $15.978 \pm 0.021$ & 200 & ST \\
\hline & 30.8272 & 1.3432 & $16.058 \pm 0.021$ & 200 & ST \\
\hline & 31.6683 & 2.1843 & $16.240 \pm 0.034$ & 100 & ST \\
\hline & 31.6933 & 2.2093 & $16.290 \pm 0.033$ & 200 & ST \\
\hline & 31.7110 & 2.227 & $16.305 \pm 0.034$ & 200 & ST \\
\hline & 31.7338 & 2.2498 & $16.342 \pm 0.031$ & 200 & ST \\
\hline & 31.7535 & 2.2695 & $16.329 \pm 0.031$ & 200 & ST \\
\hline & 31.7715 & 2.2875 & $16.326 \pm 0.033$ & 200 & ST \\
\hline & 31.7887 & 2.3047 & $16.341 \pm 0.030$ & 200 & ST \\
\hline & 31.8088 & 2.3248 & $16.351 \pm 0.030$ & 200 & ST \\
\hline & 31.8326 & 2.3486 & $16.387 \pm 0.029$ & 300 & ST \\
\hline & 31.8539 & 2.3699 & $16.411 \pm 0.028$ & 300 & ST \\
\hline & 31.8750 & 2.391 & $16.404 \pm 0.028$ & 300 & ST \\
\hline & 31.8960 & 2.412 & $16.460 \pm 0.028$ & 300 & ST \\
\hline \multirow[t]{14}{*}{2003} & April & & & & \\
\hline & 1.6086 & 3.1246 & $16.591 \pm 0.032$ & 200 & ST \\
\hline & 1.6890 & 3.205 & $16.586 \pm 0.033$ & 200 & ST \\
\hline & 1.8294 & 3.3454 & $16.639 \pm 0.029$ & 300 & ST \\
\hline & 1.8338 & 3.3498 & $16.631 \pm 0.029$ & 300 & ST \\
\hline & 2.6738 & 4.1898 & $17.211 \pm 0.031$ & 300 & ST \\
\hline & 2.7077 & 4.2237 & $17.224 \pm 0.032$ & 300 & ST \\
\hline & 2.7572 & 4.2732 & $17.254 \pm 0.035$ & 300 & ST \\
\hline & 4.6886 & 6.2046 & $17.586 \pm 0.046$ & 400 & ST \\
\hline & 4.7180 & 6.234 & $17.663 \pm 0.042$ & 400 & ST \\
\hline & 7.6029 & 9.1189 & $18.595 \pm 0.098$ & 300 & ST \\
\hline & 8.5965 & 10.1125 & $18.674 \pm 0.077$ & 400 & ST \\
\hline & 8.6405 & 10.1565 & $18.764 \pm 0.052$ & 600 & ST \\
\hline & 8.6712 & 10.1872 & $18.614 \pm 0.064$ & 400 & ST \\
\hline
\end{tabular}

